ITEP/TH-12/09

\title{
Introduction to Integral Discriminants
}

\author{
A.Morozov and Sh.Shakirov ${ }^{1}$ \\ ITEP, Moscow, Russia \\ MIPT, Dolgoprudny, Russia
}

\begin{abstract}
The simplest partition function, associated with homogeneous symmetric forms $S$ of degree $r$ in $n$ variables, is integral discriminant $J_{n \mid r}(S)=\int e^{-S\left(x_{1}, \ldots, x_{n}\right)} d x_{1} \ldots d x_{n}$. Actually, $S$-dependence remains the same if $e^{-S}$ in the integrand is substituted by arbitrary function $f(S)$, i.e. integral discriminant is a characteristic of the form $S$ itself, and not of the averaging procedure. The aim of the present paper is to calculate $J_{n \mid r}$ in a number of non-Gaussian cases. Using Ward identities - linear differential equations, satisfied by integral discriminants - we calculate $J_{2 \mid 3}, J_{2 \mid 4}, J_{2 \mid 5}$ and $J_{3 \mid 3}$. In all these examples, integral discriminant appears to be a generalized hypergeometric function. It depends on several $S L(n)$ invariants of $S$, with essential singularities controlled by the ordinary algebraic discriminant of $S$.
\end{abstract}

\section{Contents}

1 Introduction 1

2 Ward identities $\quad 3$

3 Integral Discriminants $\quad 5$

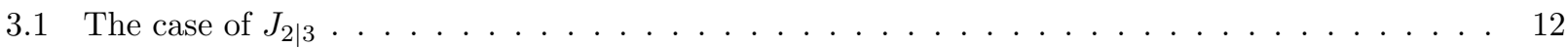

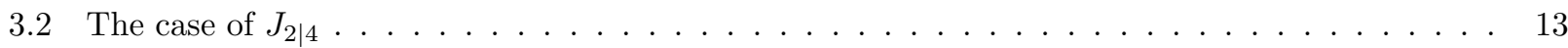



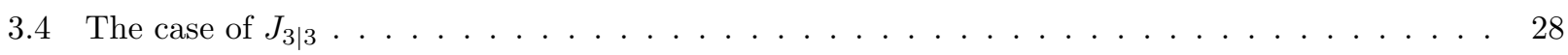

4 Conclusion $\quad 33$

\section{Introduction}

Averaging with exponential weight

$$
\langle\phi\rangle=\int \phi(x) e^{-S(x)} d x
$$

is an important operation in statistical and quantum physics. Function $S(x)$, which determines the weight, is called action. The integration domain of $x$-variables is a linear space, which is usually infinite dimensional in real physical applications: for example, a space of paths in quantum mechanics or a space of field configurations in quantum field theory. Infinite dimension of the space introduces additional complications: the integral is

${ }^{1}$ morozov@itep.ru; shakirov@itep.ru 
not always well-defined. For this reason, it is important first to study such integrals in finite dimension $n$. After this is done, one can take a $n \rightarrow \infty$ limit.

However, even in finite dimension $n$, the averaging operation is not yet fully understood. Most studied are Gaussian integrals: that is, when $S$ is quadratic in $x$-variables. The simplest Gaussian integral

$$
\langle 1\rangle=\int e^{-S_{i j} x_{i} x_{j}} d^{n} x
$$

is easily calculated and expressed through an invariant quantity - determinant - e.g. by diagonalising $S$ :

$$
\int e^{-S_{i j} x_{i} x_{j}} d^{n} x=\int e^{-\lambda_{1} x_{1}^{2}-\ldots-\lambda_{n} x_{n}^{2}} d^{n} x=\frac{1}{\sqrt{\lambda_{1} \ldots \lambda_{n}}} \int e^{-x_{1}^{2}-\ldots-x_{n}^{2}} d^{n} x \sim \frac{1}{\sqrt{\operatorname{det} S}}
$$

The integral which factors out is just an $S$-independent constant, which can be finite or infinite depending on the contour of integration. Therefore, essential $S$-dependence of this Gaussian integral is given by $(\operatorname{det} S)^{-1 / 2}$. Any other Gaussian integral - with non-homogeneous quadratic $S$ or non-trivial $\phi$ - is equally easy, because we have enough freedom to transform quadratic $S$ to diagonal or any other desired form. The possibility of diagonalisation greatly simplifies calculations with matrices (tensors with two indices).

Unfortunately, such methods do not work when $S$ is cubic or higher degree. This can be seen already from dimension counting. The number of independent coefficients $S_{i j k}$, which is $n(n+1)(n+2) / 6$, exceeds the number $n^{2}-1$ of available $S L(n)$ transformations, so it is not generally possible to diagonalize a cubic action - the group $S L(n)$ is too small. That is why the integral

$$
\int e^{-S_{i j k} x_{i} x_{j} x_{k}} d^{n} x
$$

and its higher degree analogues

$$
J_{n \mid r}=\int e^{-S\left(x_{1}, \ldots, x_{n}\right)} d^{n} x, \quad S\left(x_{1}, \ldots, x_{n}\right)=S_{i_{1}, \ldots, i_{r}} x_{i_{1}} \ldots x_{i_{r}}
$$

despite simply-looking, still remain terra incognita. In $[1,2]$ integral $J_{n \mid r}(S)$ was named the integral discriminant of $S$, because in the simplest cases this integral is just a power of algebraic discriminant $D_{n \mid r}(S)$. For example, there is an inspiring formula for 3 -forms in two variables

$$
J_{2 \mid 3}=\int e^{-\left(a x^{3}+b x^{2} y+c x y^{2}+d y^{3}\right)} d x d y=\left(27 a^{2} d^{2}-b^{2} c^{2}-18 a b c d+4 a c^{3}+4 b^{3} d\right)^{-1 / 6}=\left(D_{2 \mid 3}\right)^{-1 / 6}
$$

which shows that $J_{2 \mid 3}$ and $D_{2 \mid 3}$ are, indeed, related. However, when one goes to higher $n$ and $r$, the relation gets more complicated: $D_{n \mid r}$ defines only the singularities of $J_{n \mid r}$, while non-singular behaviour is controlled by other algebraic invariants. Thus, theory of integral discriminants is closely connected to invariant theory [3] and can be viewed as one of the branches of non-linear algebra [2], [4] - [8].

It would be very interesting to find a closed formula for generic $J_{n \mid r}$, because it could provide exciting new tools in QFT, statistics and other fields where non-Gaussian averaging is used. In this paper, we make a step in this direction and find integral discriminants explicitly for 3-forms, 4-forms and 5-forms in two variables, and for 3 -forms in three variables. Our results indicate an intriguing connection between integral discriminants (1) and special functions known as generalized hypergeometric functions [9]. 


\section{$2 \quad$ Ward identities}

When the action is non-quadratic, diagonalisation and similar linear-algebra tricks fail. To handle nonGaussian integrals, one needs essentially different methods. One of such methods (actually originated in the context of quantum field theory) is to find a differential equation, satisfied by the integral as a function of its parameters, see $[10,11]$ for typical applications and references. If such differential equation exists, we call it Ward identity (even if it is not directly induced by a change of integration variables).

Since Ward identities play the central role in present paper, let us give a pair of simple examples to clarify this issue. An integral

$$
F(a)=\int e^{-x^{2} / 2+a x} d x
$$

satisfies a Ward identity

$$
\left(a-\frac{\partial}{\partial a}\right) F(a)=0
$$

because

$$
\left(a-\frac{\partial}{\partial a}\right) F(a)=\int(a-x) e^{-x^{2} / 2+a x} d x=\int\left(\frac{\partial}{\partial x} e^{-x^{2} / 2+a x}\right) d x=0
$$

In contrast with diagonalisation, this method is perfectly generalisable to non-Gaussian integrals: say,

$$
G(a)=\int e^{-x^{3} / 3+a x} d x
$$

satisfies a Ward identity

$$
\left(a-\frac{\partial^{2}}{\partial a^{2}}\right) G(a)=0
$$

because

$$
\left(a-\frac{\partial^{2}}{\partial a^{2}}\right) G(a)=\int\left(a-x^{2}\right) e^{-x^{3} / 3+a x} d x=\int\left(\frac{\partial}{\partial x} e^{-x^{3} / 3+a x}\right) d x=0
$$

In this way the problem of non-Gaussian integrals is reduced to another problem - of differential equations. This is a much easier problem, especially if differential equations are linear. In the first case we have

$$
a F(a)=\frac{\partial}{\partial a} F(a) \Longrightarrow F(a)=c \cdot e^{a^{2} / 2}
$$

while in the second case

$$
a G(a)=\frac{\partial^{2}}{\partial a^{2}} G(a) \Longrightarrow G(a)=c_{1} \cdot \operatorname{Ai}(a)+c_{2} \cdot \operatorname{Bi}(a)
$$

where $\mathrm{Ai}$ and $\mathrm{Bi}$ are special functions - Airy functions of the first and second kind. Note that, there is only 


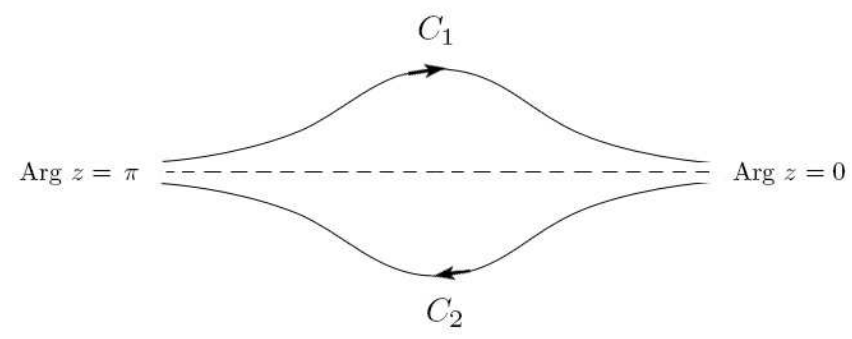

Figure 1: Two, out of infinitely many, admissible contours of integration for the integral $\int e^{-x^{2} / 2+a x} d x$.

one linear independent solution in the Gaussian case, while in the non-Gaussian case there are two linearindependent solutions. This is because to correctly define an integral, one still needs to specify an integration contour. Different integration contours provide different solutions of the Ward identity.

This relationship between Ward identities and integration contours is quite important, so let us add more details. As the simplest option, the contour of integration in the integral

$$
F(a)=\int_{C} e^{-x^{2} / 2+a x} d x
$$

can be chosen as $C=$ real axis. However, this choice is by no means unique. In fact, any other contour $C$ which asymptotically tends to the lines $\operatorname{Arg} z=0$ and $\operatorname{Arg} z=\pi / 2$, is admissible. A contour $C$ is said to be admissible, if the integral over $C$ converges. A few admissible contours are shown at Fig. 1. Note that, to ensure vanishing of the integral of full derivative (and thus validity of Ward identities) we consider only closed contors - the contours at Fig. 1. are closed on the Riemann sphere, if the infinitely remote point is taken into account. Since Ward identity in this case is a differential equation of first order, all the contours give one and the same answer $F(a)=\exp \left(a^{2} / 2\right)$ up to proportionality.

More interesting is the non-Gaussian integral

$$
G(a)=\int_{C} e^{-x^{3} / 3+a x} d x
$$

Note that, the real axis is no longer an admissible contour, since $\exp \left(-x^{3}\right)$ grows to infinity when $x \rightarrow-\infty$. In this case, admissible is any contour $C$ which asymptotically tends to the lines $\operatorname{Arg} z=0, \operatorname{Arg} z=2 \pi / 3$ and $\operatorname{Arg} z=4 \pi / 3$. A few admissible contours are shown at Fig. 2. Again, to ensure vanishing of the integral of full derivative, we consider only closed contors. Since Ward identity is second order in this case, there are two essentially different integration contours, say, $C_{1}$ and $C_{2}$. An integral over arbitrary contour - the general solution of the Ward identity - is given by linear combination

$$
\int e^{-x^{3} / 3+a x} d x=c_{1} \int_{C_{1}} e^{-x^{3} / 3+a x} d x+c_{2} \int_{C_{2}} e^{-x^{3} / 3+a x} d x
$$

To summarize the above examples, Ward identity is the main differential equation which governs all the contours at once. The choice of particular contour corresponds to the choice of particular solution of the Ward identity. For this reason, Ward identities are especially convenient to study of properties, which are invariant under change of integration contour. Additional details can be found in [10]. 


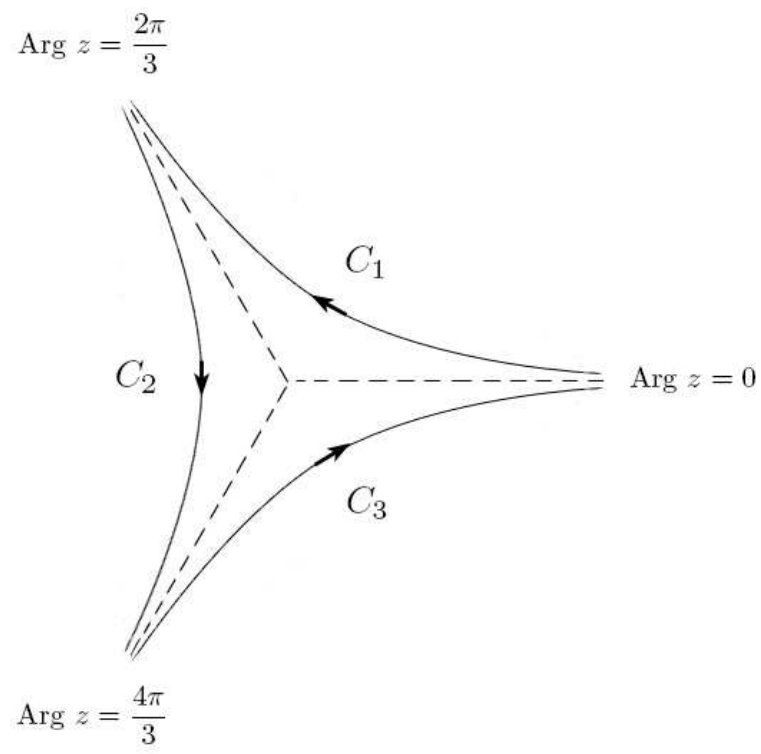

Figure 2: Three, out of infinitely many, admissible contours of integration for the integral $\int e^{-x^{3} / 3+a x} d x$.

\section{$3 \quad$ Integral Discriminants}

Definition. In this paper we study a specific class of non-Gaussian integrals: integral discriminants

$$
J_{n \mid r}(S)=\int e^{-S\left(x_{1}, x_{2}, \ldots, x_{n}\right)} d x_{1} \ldots d x_{n}
$$

associated with homogeneous symmetric $r$-forms $S\left(x_{1}, x_{2}, \ldots, x_{n}\right)$. There are two different notations for symmetric forms, which can be useful under different circumstances: tensor notation

$$
S\left(x_{1}, x_{2}, \ldots, x_{n}\right)=\sum_{i_{1}, i_{2}, \ldots, i_{r}=1}^{n} S_{i_{1}, i_{2}, \ldots, i_{r}} x_{i_{1}} x_{i_{2}} \ldots x_{i_{r}}
$$

and monomial notation

$$
S\left(x_{1}, x_{2}, \ldots, x_{n}\right)=\sum_{a_{1}+a_{2}+\ldots+a_{n}=r} s_{a_{1}, a_{2} \ldots a_{n}} x_{1}^{a_{1}} x_{2}^{a_{2}} \ldots x_{n}^{a_{n}}
$$

To distinguish between these notations, we denote coefficients by capital and small letters, respectively. Note, that in tensor notation coefficients have $r$ indices, while in monomial notation they have $n$ indices.

The choice of contour. Being non-Gaussian integrals, integral discriminants of course depend on the choice of integration contour. One has to remember, that not every contour is admissible: for given $S$, only restricted set of contours is allowed. Say, for positive-definite quadratic forms $S$ admissible is any contour, which is asymptotically pure real (see the previous section, especially Fig. 1 and Fig. 2 for simple examples). At the same time for negative-definite quadratic forms admissible is any contour, which is asymptotically pure imaginary. In this paper, we do not describe the contour dependence explicitly. Instead, we concentrate on contour-independent properties of integral discriminants. 
Independence on the form of action. An important feature of integral discriminants, which is due to homogeneity of $S$, is the possibility to substitute the function $e^{-S}$ under the integral with arbitrary function (or, better to say, arbitrary good function) $f(S)$ :

$$
\int e^{-S\left(x_{1}, \ldots, x_{n}\right)} d x_{1} \ldots d x_{n} \sim \int f\left(S\left(x_{1}, \ldots, x_{n}\right)\right) d x_{1} \ldots d x_{n}
$$

Even before specifying the class of good functions, let us consider a simple illustration with $f(S)=e^{-S^{2}}$ :

$$
\begin{gathered}
\int e^{-\left(S_{i j} x_{i} x_{j}\right)} d^{n} x=\int e^{-\left(\lambda_{1} x_{1}^{2}+\ldots+\lambda_{n} x_{n}^{2}\right)} d^{n} x=\frac{1}{\sqrt{\lambda_{1} \ldots \lambda_{n}}} \int e^{-\left(x_{1}^{2}+\ldots+x_{n}^{2}\right)} d^{n} x=\frac{\text { const }}{\sqrt{\operatorname{det} S}} \\
\int e^{-\left(S_{i j} x_{i} x_{j}\right)^{2}} d^{n} x=\int e^{-\left(\lambda_{1} x_{1}^{2}+\ldots+\lambda_{n} x_{n}^{2}\right)^{2}} d^{n} x=\frac{1}{\sqrt{\lambda_{1} \ldots \lambda_{n}}} \int e^{-\left(x_{1}^{2}+\ldots+x_{n}^{2}\right)^{2}} d^{n} x=\frac{\text { const }^{\prime}}{\sqrt{\operatorname{det} S}}
\end{gathered}
$$

To specify the class of good functions and prove (2), let us make a change of integration variables

$$
x_{1}=\rho, \quad x_{2}=\rho z_{2}, \quad x_{3}=\rho z_{3}, \ldots, x_{n}=\rho z_{n}
$$

i.e. pass from homogeneous coordinates $x_{i}$ to non-homogeneous coordinates $z_{i}=x_{i} / x_{1}$. Then

$$
\begin{gathered}
\int e^{-S\left(x_{1}, x_{2}, \ldots, x_{n}\right)} d x_{1} \ldots d x_{n}=\int \rho^{n-1} d \rho \int d z_{2} \ldots d z_{n} e^{-\rho^{r} S\left(1, z_{2}, \ldots, z_{n}\right)}= \\
=\left(\int \rho^{n-1} e^{-\rho^{r}} d \rho\right) \cdot \int \frac{d z_{2} \ldots d z_{n}}{S\left(1, z_{2}, \ldots, z_{n}\right)^{n / r}}
\end{gathered}
$$

For the right hand side of (2) we have

$$
\begin{gathered}
\int f\left(S\left(x_{1}, x_{2}, \ldots, x_{n}\right)\right) d x_{1} \ldots d x_{n}=\int \rho^{n-1} d \rho \int d z_{2} \ldots d z_{n} f\left(\rho^{r} S\left(1, z_{2}, \ldots, z_{n}\right)\right)= \\
=\left(\int \rho^{n-1} f\left(\rho^{r}\right) d \rho\right) \cdot \int \frac{d z_{2} \ldots d z_{n}}{S\left(1, z_{2}, \ldots, z_{n}\right)^{n / r}}
\end{gathered}
$$

Both integrals over $\rho$ are just $S$-independent constants. As one can see, (2) is valid, iff these integrals

$$
\int \rho^{n-1} e^{-\rho^{r}} d \rho \quad \text { and } \quad \int \rho^{n-1} f\left(\rho^{r}\right) d \rho
$$

are finite over one and the same contour. This condition specifies the class of good functions $f(S)$. For example, all functions $f(S)=\exp \left(-S^{k}\right)$ for $k>0$ fall into this class. Relation (2) is therefore proved. As a byproduct, we have obtained a non-homogeneous integral representation

$$
J_{n \mid r}(S)=\text { const } \cdot \int \frac{d z_{2} \ldots d z_{n}}{S\left(1, z_{2}, \ldots, z_{n}\right)^{n / r}}
$$


A useful complement to the definiton of integral discriminants, representation (3) highlights two properties, which were less evident in (1): the scaling dimension

$$
J_{n \mid r}(\lambda S)=\lambda^{-n / r} J_{n \mid r}(S)
$$

and the vertical symmetry

$$
I_{n \mid k r}\left(S^{k}\right) \sim J_{n \mid r}(S)
$$

Ward identities. We now turn to Ward identities, satisfied by integral discriminants with respect to their parameters. Let us introduce the correlation functions

$$
\left\langle\phi\left(x_{1}, \ldots, x_{n}\right)\right\rangle=\int \phi\left(x_{1}, \ldots, x_{n}\right) e^{-S\left(x_{1}, x_{2}, \ldots, x_{n}\right)} d x_{1} \ldots d x_{n}
$$

These correlation functions satisfy

$$
\frac{\partial}{\partial s_{a_{1}, \ldots, a_{n}}}\left\langle\phi\left(x_{1}, \ldots, x_{n}\right)\right\rangle=-\left\langle x_{1}^{a_{1}} \ldots x_{n}^{a_{n}} \phi\left(x_{1}, \ldots, x_{n}\right)\right\rangle
$$

and

$$
\frac{\partial}{\partial s_{a_{1}, \ldots, a_{n}}} \frac{\partial}{\partial s_{b_{1}, \ldots, b_{n}}}\left\langle\phi\left(x_{1}, \ldots, x_{n}\right)\right\rangle=\left\langle x_{1}^{a_{1}+b_{1}} \ldots x_{n}^{a_{n}+b_{n}} \phi\left(x_{1}, \ldots, x_{n}\right)\right\rangle
$$

The right hand side depends only on the sum of indices $a_{i}+b_{i}$, not on $a_{i}$ and $b_{i}$ separately. For this reason, correlation functions satisfy a system of homogeneous second order differential equations:

$$
\left(\frac{\partial}{\partial s_{\vec{a}}} \frac{\partial}{\partial s_{\vec{b}}}-\frac{\partial}{\partial s_{\vec{p}}} \frac{\partial}{\partial s_{\vec{q}}}\right)\left\langle\phi\left(x_{1}, \ldots, x_{n}\right)\right\rangle=0, \quad \vec{a}+\vec{b}=\vec{p}+\vec{q}
$$

In particular, if we set $\phi\left(x_{1}, \ldots, x_{n}\right)=1$ we find that integral discriminant $\langle 1\rangle=J_{n \mid r}$ satisfies Ward identities

$$
\left(\frac{\partial}{\partial s_{\vec{a}}} \frac{\partial}{\partial s_{\vec{b}}}-\frac{\partial}{\partial s_{\vec{p}}} \frac{\partial}{\partial s_{\vec{q}}}\right) J_{n \mid r}(S)=0, \quad \vec{a}+\vec{b}=\vec{p}+\vec{q}
$$

These equations do not exhaust the set of all Ward identities: there are more. Notice that the differential operator in the left hand side of (8) annihilates not only the integral, but also the integrand:

$$
\left(\frac{\partial}{\partial s_{\vec{a}}} \frac{\partial}{\partial s_{\vec{b}}}-\frac{\partial}{\partial s_{\vec{p}}} \frac{\partial}{\partial s_{\vec{q}}}\right) e^{-S\left(x_{1}, x_{2}, \ldots, x_{n}\right)}=0, \quad \vec{a}+\vec{b}=\vec{p}+\vec{q}
$$

and even

$$
\left(\frac{\partial}{\partial s_{\vec{a}}} \frac{\partial}{\partial s_{\vec{b}}}-\frac{\partial}{\partial s_{\vec{p}}} \frac{\partial}{\partial s_{\vec{q}}}\right) f\left(S\left(x_{1}, \ldots, x_{n}\right)\right)=0, \quad \vec{a}+\vec{b}=\vec{p}+\vec{q}
$$


which justifies (2) once again. Usually, Ward differential operators do not annihilate the integrand, they just transform the integrand into a full derivative (which implies vanishing of the integral over any closed contour). Differential operator in the left hand side of (8) is therefore too special; one can expect other Ward identities to exist. To find them, let us consider the most general vanishing correlator - an integral of full derivative

$$
\int \frac{\partial}{\partial x_{i}}\left(\phi\left(x_{1}, \ldots, x_{n}\right) e^{-S\left(x_{1}, x_{2}, \ldots, x_{n}\right)}\right) d x_{1} \ldots d x_{n}=0
$$

Taking the derivative, we obtain Ward identities for correlation functions:

$$
\left\langle\frac{\partial \phi\left(x_{1}, \ldots, x_{n}\right)}{\partial x_{i}}\right\rangle=\left\langle\phi\left(x_{1}, \ldots, x_{n}\right) \frac{\partial S\left(x_{1}, \ldots, x_{n}\right)}{\partial x_{i}}\right\rangle
$$

For now we are not interested in all correlation functions, only in the integral discriminant. We need to rewrite the equations (9) as differential equations on $J_{n \mid r}$. There are several ways to do this, the simplest way is to set $\phi\left(x_{1}, \ldots, x_{n}\right)=x_{j}$ and use the identity (6) to get rid of remaining correlators. Doing so, we obtain equations

$$
\left(\delta_{i j}+a_{i} \hat{A}_{i j}\right) J_{n \mid r}=0
$$

where operators

$$
\hat{A}_{i j}=\sum_{a_{1}+a_{2}+\ldots+a_{n}=r} s_{a_{1} \ldots a_{i}-1 \ldots a_{j}+1 \ldots a_{n}} \frac{\partial}{\partial s_{a_{1} \ldots a_{n}}}
$$

form the $G L(n)$ algrebra:

$$
\hat{A}_{i j} \hat{A}_{k l}-\hat{A}_{k l} \hat{A}_{i j}=\hat{A}_{i l} \delta_{k j}-\hat{A}_{k j} \delta_{i l}
$$

Note also, that other choices of $\phi\left(x_{1}, \ldots, x_{n}\right)$ in (9) do not give new Ward identities: everything, that can be obtained in this way, will be equivalent to (10). We conclude that the complete set of Ward identities for $J_{n \mid r}$ consists of equations (8) and (10).

$S L(n)$ invariance. In solving these Ward identities we start from (10), because they are first order. In fact, equations (10) simply reflect the $G L(n)$-covariance $(=S L(n)$ invariance + correct scaling) of the model. This becomes clear, if we separate the generators of $G L(n)$ into two parts: the dilatation (degree) operator

$$
\hat{A}_{11}+\ldots+\hat{A}_{n n}
$$

and the other $n^{2}-1$ operators, which form a representation of $S L(n)$. The first relation implies

$$
\left(\hat{A}_{11}+\ldots+\hat{A}_{n n}\right) J_{n \mid r}=-\frac{n}{r} J_{n \mid r}
$$

which is nothing but the scaling dimension (4). The other $n^{2}-1$ relations imply, that $J_{n \mid r}$ is $S L(n)$-invariant 
function of $S$. Thus, equations (10) are completely solved by any $S L(n)$-invariant function of $S$, which is in addition homogeneous in $S$ of degree $-n / r$. A natural question is: is there any simple description of such functions? Actually, the answer to this question is positive: any $S L(n)$-invariant function can be uniqely represented as a function of the elementary invariants $I_{k}$

$$
J_{n \mid r}=F\left\{I_{k}\right\}
$$

much in the same way as any $S L(n)$-invariant function of a matrix $A_{j}^{i}$ can be uniquely represented as a function of elementary invariants $\operatorname{tr} A^{k}$. Unfortunately, in the case of symmetric tensors $S_{i_{1}, \ldots, i_{r}}$ classification of these elementary invariants $I_{k}$ is not that easy, as in the case of matrices. Since a symmetric tensor with $r$ indices is much more complicated, than a matrix with two indices, no explicit formula like $I_{k}=\operatorname{tr} A^{k}$ is available. The study of properties of the elementary invariants $I_{k}$, of different kinds of explicit formulas and relations between them, is a classical branch of science known as invariant theory [3].

The number of $S L(n)$ invariants. Given parameters $n$ and $r$ of the form $S$, one can easily find the number of elementary invariants $I_{k}$, of which all other invariants are various functions. Indeed, the linear space of forms $S$ of type $n \mid r$ has dimension

$$
\operatorname{dim} S_{n \mid r}=\frac{(n+r-1) !}{r !(n-1) !}
$$

that is the number of independent coefficients of symmetric tensor $S_{i_{1} \ldots i_{r}}$. The group $S L(n)$ acts on this space, dividing it into orbits. All forms, connected by $S L(n)$ transformations $S_{i_{1} \ldots i_{r}} \mapsto S_{i_{1} \ldots i_{r}} U_{j_{1}}^{i_{1}} \ldots U_{j_{n}}^{i_{n}}, \quad U \in S L(n)$ belong to one orbit. Ward identities imply, that $J_{n \mid r}$ does not depend on coordinates along the orbit ("angular" variables). It depends only on transverse coordinates which label orbits ("radial" variables). Simple counting of dimensions implies, that dimension of the space of orbits, i.e. the number of radial coordinates, equals to

$$
\operatorname{dim} S_{n \mid r}-\operatorname{dim} S L(n)=\frac{(n+r-1) !}{r !(n-1) !}-n^{2}+1
$$

Several examples of these numbers are shown at Fig. 3.

\begin{tabular}{c|cccccc}
$r \backslash n$ & 2 & 3 & 4 & 5 & 6 & 7 \\
\hline 2 & 1 & 1 & 1 & 1 & 1 & 1 \\
3 & 1 & 2 & 5 & 11 & 21 & 36 \\
4 & 2 & 7 & 20 & 46 & 91 & 162 \\
5 & 3 & 13 & 41 & 102 & 217 & 414 \\
6 & 4 & 20 & 69 & 186 & 427 & 876
\end{tabular}

Figure 3: The number of functionally independent $S L(n)$ invariants $I_{k}$ of a form of degree $r$ in $n$ variables. 
The Gaussian case $J_{n \mid 2}$. Note, however, that the case $r=2$ (quadratic forms) is exceptional, because the dimension $\operatorname{dim} S_{n \mid 2}=n(n+1) / 2$ is less than the dimension $\operatorname{dim} S L(n)=n^{2}-1$. For this reason, the above dimension counting does not work in this (and only in this) case. Actually, as we know, the space of $S L(n)$ orbits on quadratic forms is one-dimensional, and the only invariant - the single coordinate on the space of orbits - is determinant $\operatorname{det} S$. Thus, determinant is the only variable $J_{n \mid 2}$ can depend on:

$$
J_{n \mid 2}(S)=F(\operatorname{det} S)
$$

The homogeneity condition states, that $F(\lambda x)=F(x) / \sqrt{\lambda}$ and has a single solution $F(x)=1 / \sqrt{x}$. In this way we reproduce the well-known Gaussian integral

$$
J_{n \mid 2}(S)=\frac{1}{\sqrt{\operatorname{det} S}}
$$

We emphasize, that simplicity of this answer is due to simplicity of the space of orbits, i.e, due to the fact there is a single invariant in this case. For higher $r$, there are many invariants (as many as shown at Fig. 3) and $J_{n \mid r}$ is a non-trivial function of all of them. The problem is that it becomes impossible to find $J_{n \mid r}$ from the homogeneity condition alone. The solution of this problem is provided by Ward identities: in addition to the homogeneity condition, integral discriminant satisfies relations (8) and this allows to find $F\left\{I_{k}\right\}$.

Diagram technique for $S L(n)$ invariants. To do actual computations with $S L(n)$ invariants, we will adopt a convenient diagram technique, which is described in [2]. According to [2], it is possible to represent tensors with $k$ indices as $k$-valent vertices, with two types of indices - covariant and contravariant - represented by two types of lines. Contraction of indices is naturally represented as connection of these lines. To simplify the diagrams, we use solid lines for both covariant and contravariant indices. In this paper we use the following four elementary building blocks for diagrams: covariant tensor $S_{i_{1} \ldots i_{r}}$, represented by a black $r$-valent vertex



contravariant tensor $\frac{\partial}{\partial S_{i_{1} \ldots i_{r}}}$, represented by a black $r$-valent vertex

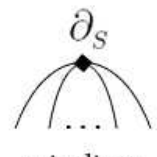

$r$ indices

completely antisymmetric contravariant tensor $\epsilon^{i_{1} \ldots i_{n}}$, represented by a white $n$-valent vertex 


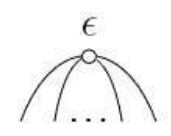

$n$ indices

and completely antisymmetric covariant tensor $\epsilon_{i_{1} \ldots i_{n}}^{*}$, represented by a white $n$-valent vertex

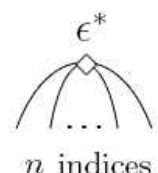

Tensors $\epsilon^{i_{1} \ldots i_{n}}$ and $\epsilon_{i_{1} \ldots i_{n}}^{*}$ are completely antisymmetric with respect to permutations of their indices, and, therefore, remain invariant under $S L(n)$ transformations:

$$
\begin{aligned}
& \epsilon^{i_{1} \ldots i_{n}} U_{i_{1}}^{j_{1}} \ldots U_{i_{n}}^{j_{n}}=\operatorname{det} U \cdot \epsilon^{j_{1} \ldots j_{n}}=\epsilon^{j_{1} \ldots j_{n}} \\
& \epsilon_{i_{1} \ldots i_{n}}^{*} U_{j_{1}}^{i_{1}} \ldots U_{j_{n}}^{i_{n}}=\operatorname{det} U \cdot \epsilon_{j_{1} \ldots j_{n}}^{*}=\epsilon_{j_{1} \ldots j_{n}}^{*}
\end{aligned}
$$

where $\operatorname{det} U=1$, because $U \in S L(n)$. For this reason, any diagram without free (uncontracted) indices, made of contravariant $\epsilon$-vertices and covariant $S$-vertices, is automatically $S L(n)$-invariant function of $S$. Say, determinant of $n \times n$ matrix $S_{i j}$ can be represented as a diagram at Fig. 4 , with $n S$-vertices and two $\epsilon$-vertices. The other two types of vertices $\left(\partial / \partial S\right.$ and $\left.\epsilon^{*}\right)$ will be used to construct $S L(n)$-invariant differential operators.

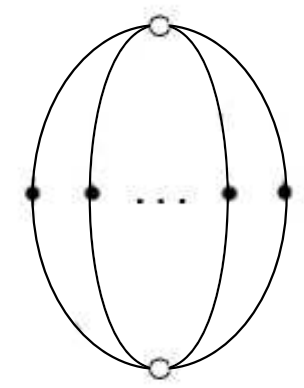

Figure 4: Determinant of matrix $S_{i j}$, represented as a diagram of tensor contraction. Black 2-valent vertices represent tensor $S$, white n-valent vertices represent tensor $\epsilon$.

Diagrams provide a convenient way to obtain $S L(n)$ invariants. In fact, any $S L(n)$-invariant can be obtained in this way, but, unfortunately, not uniquely: absolutely different-looking diagrams can represent one and the same invariant. Trying to resolve this ambiguity, one typically faces the complicated problems of classification of diagrams and finding relations between diagrams. These problems will not be adressed (and even touched) in present paper. Our goal is different: to find an explicit answer for $J_{n \mid r}$ in several non-Gaussian cases, using diagrams as a convenient tool. We now turn to accomplishing this goal. 


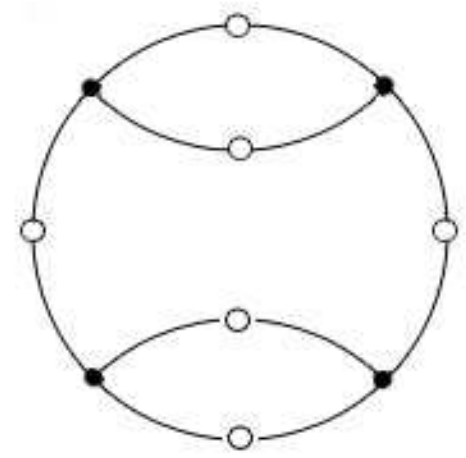

Figure 5: The degree 4 invariant $I_{4}$ of a 3 -form in 2 variables, represented as a diagram of tensor contraction. Black 3-valent vertices represent tensor $S$, white 2-valent vertices represent tensor $\epsilon$.

\subsection{The case of $J_{2 \mid 3}$}

The simplest non-trivial (i.e. non-Gaussian) example is a 3 -form in 2 variables, which can be written as

$$
S(x, y)=S_{111} x^{3}+3 S_{112} x^{2} y+3 S_{122} x y^{2}+S_{222} y^{2}
$$

By dimension counting, there is only one elementary invariant $I_{4}$ in this case, given by a diagram at Fig. 5 . The subscript "4" stands for the degree of this invariant. In this paper we find it convenient to denote the elementary invariants of degree $k$ as $I_{k}$. It is straightforward to write the algebraic expression for the diagram:

$$
I_{4}=S_{i_{1} i_{2} i_{3}} S_{j_{1} j_{2} j_{3}} S_{k_{1} k_{2} k_{3}} S_{l_{1} l_{2} l_{3}} \epsilon^{i_{1} j_{1}} \epsilon^{i_{2} j_{2}} \epsilon^{k_{1} l_{1}} \epsilon^{k_{2} l_{2}} \epsilon^{i_{3} k_{3}} \epsilon^{j_{3} l_{3}}
$$

Evaluating this sum, one gets the following explicit formula for $I_{4}$

$$
I_{4}=2 S_{111}^{2} S_{222}^{2}-12 S_{111} S_{112} S_{122} S_{222}+8 S_{111} S_{122}^{3}+8 S_{112}^{3} S_{222}-6 S_{112}^{2} S_{122}^{2}
$$

which is nothing but the algebraic discriminant $D_{2 \mid 3}$ of $S$ :

$$
D_{2 \mid 3}=I_{4}
$$

Since there is only one elementary invariant, the integral discriminant $J_{2 \mid 3}$ must be a function of $D_{2 \mid 3}$ :

$$
J_{2 \mid 3}(S)=F\left(I_{4}\right)
$$

Thus, this case is similar to the Gaussian case. The homogeneity condition states, that $F(\lambda x)=F(x) / \sqrt[6]{\lambda}$ and has a single solution $F(x)=1 / \sqrt[6]{x}$. In this way we reproduce the formula from the Introduction:

$$
J_{2 \mid 3}(S)=I_{4}^{-1 / 6}
$$

In this case, one does not need to use the Ward identities (8): it is enough to use the homogeneity condition. 


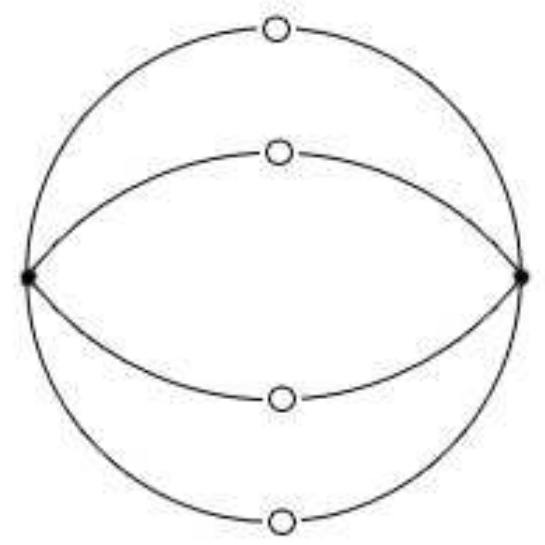

Figure 6: The degree 2 invariant $I_{2}$ of a 4 -form in 2 variables, represented as a diagram of tensor contraction. Black 4-valent vertices represent tensor $S$, white 2-valent vertices represent tensor $\epsilon$.

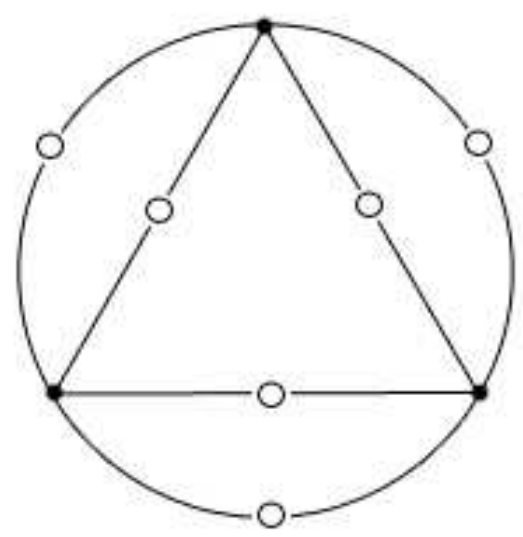

Figure 7: The degree 3 invariant $I_{3}$ of a 4 -form in 2 variables, represented as a diagram of tensor contraction. Black 4-valent vertices represent tensor $S$, white 2 -valent vertices represent tensor $\epsilon$.

\subsection{The case of $J_{2 \mid 4}$}

The next-to-simplest example is a 4 -form in 2 variables, which can be written as

$$
S(x, y)=S_{1111} x^{4}+4 S_{1112} x^{3} y+6 S_{1122} x^{2} y^{2}+4 S_{1222} x y^{3}+S_{2222} y^{4}
$$

Invariants. By dimension counting, there are two elementary invariants in this case. They have relatively low degrees 2 and 3, denoted as $I_{2}$ and $I_{3}$ and given by diagrams at Fig. 6 and Fig. 7, respectively. Looking at the diagrams, it is straightforward to write algebraic expressions for $I_{2}, I_{3}$ :

$$
\begin{gathered}
I_{2}=S_{i_{1} i_{2} i_{3} i_{4}} S_{j_{1} j_{2} j_{3} j_{4}} \epsilon^{i_{1} j_{1}} \epsilon^{i_{2} j_{2}} \epsilon^{i_{3} j_{3}} \epsilon^{i_{4} j_{4}} \\
I_{3}=S_{i_{1} i_{2} i_{3} i_{4}} S_{j_{1} j_{2} j_{3} j_{4}} S_{k_{1} k_{2} k_{3} k_{4}} \epsilon^{i_{1} j_{1}} \epsilon^{i_{2} j_{2}} \epsilon^{i_{3} k_{1}} \epsilon^{i_{4} k_{2}} \epsilon^{j_{3} k_{3}} \epsilon^{j_{4} k_{4}}
\end{gathered}
$$


Evaluating these sums, one gets the following explicit formulas for $I_{2}, I_{3}$ :

$$
\begin{gathered}
I_{2}=2 S_{1111} S_{2222}-8 S_{1112} S_{1222}+6 S_{1122}^{2} \\
I_{3}=6 S_{1111} S_{1122} S_{2222}-6 S_{1111} S_{1222}^{2}-6 S_{1112}^{2} S_{2222}+12 S_{1112} S_{1122} S_{1222}-6 S_{1122}^{3}
\end{gathered}
$$

The algebraic discriminant $D_{2 \mid 4}$, just like any other $S L(2)$-invariant function of $S$, is a function of $I_{2}, I_{3}$ :

$$
\begin{aligned}
D_{2 \mid 4}= & I_{2}^{3}-6 I_{3}^{2}=8 S_{1111}^{3} S_{2222}^{3}-96 S_{1111}^{2} S_{1112} S_{1222} S_{2222}^{2}-144 S_{1111}^{2} S_{1122}^{2} S_{2222}^{2}+432 S_{1111}^{2} S_{1122} S_{1222}^{2} S_{2222} \\
& -216 S_{1111}^{2} S_{1222}^{4}+432 S_{1111} S_{1112}^{2} S_{1122} S_{2222}^{2}-48 S_{1111} S_{1112}^{2} S_{1222}^{2} S_{2222}-1440 S_{1111} S_{1112} S_{1122}^{2} S_{1222} S_{2222} \\
& +648 S_{1111} S_{1122}^{4} S_{2222}+864 S_{1111} S_{1112} S_{1122} S_{1222}^{3}+864 S_{1112}^{3} S_{1122} S_{1222} S_{2222}+288 S_{1112}^{2} S_{1122}^{2} S_{1222}^{2} \\
& -432 S_{1111} S_{1122}^{3} S_{1222}^{2}-216 S_{1112}^{4} S_{2222}^{2}-512 S_{1112}^{3} S_{1222}^{3}-432 S_{1112}^{2} S_{1122}^{3} S_{2222}
\end{aligned}
$$

Integral discriminant. Similarly, the integral discriminant is a function of $I_{2}, I_{3}$ :

$$
J_{2 \mid 4}=F\left(I_{2}, I_{3}\right)
$$

where the function $F$ is to be determined. The homogeneity condition does not allow to find $F$ unambigously, it constrains only the overall scaling factor, but not the non-trivial dependence on the ratio of invariants:

$$
F\left(I_{2}, I_{3}\right)=I_{2}^{-1 / 4} G\left(\frac{I_{3}^{2}}{I_{2}^{3}}\right)
$$

Ward identities. To find the function $G(z)$ in this ansatz, we need to use the Ward identities (8). Applied to the present case of $n=2, r=4$, the system (8) takes the form

$$
\begin{aligned}
& \left(\frac{\partial^{2}}{\partial s_{40} \partial s_{22}}-\frac{\partial^{2}}{\partial s_{31} \partial s_{31}}\right) J_{2 \mid 4}=0 \\
& \left(\frac{\partial^{2}}{\partial s_{40} \partial s_{13}}-\frac{\partial^{2}}{\partial s_{31} \partial s_{22}}\right) J_{2 \mid 4}=0 \\
& \left(\frac{\partial^{2}}{\partial s_{40} \partial s_{04}}-\frac{\partial^{2}}{\partial s_{31} \partial s_{13}}\right) J_{2 \mid 4}=0 \\
& \left(\frac{\partial^{2}}{\partial s_{22} \partial s_{22}}-\frac{\partial^{2}}{\partial s_{31} \partial s_{13}}\right) J_{2 \mid 4}=0
\end{aligned}
$$

where $s$-parameters and $S$-parameters are related by

$$
s_{40}=S_{1111}, s_{31}=4 S_{1112}, s_{22}=6 S_{1122}, s_{13}=4 S_{1222}, s_{04}=S_{2222}
$$

Particular equations in this system are, of course, not $S L(2)$ invariant. This is not convenient, since we are interested in $S L(2)$-invariant solutions. Let us transform Ward identities into invariant form, using (16). 
Invariant form of Ward identities. Substituting the invariant anzatz (16) into the system, we obtain

$$
\begin{gathered}
\left(\frac{\partial^{2}}{\partial s_{40} \partial s_{22}}-\frac{\partial^{2}}{\partial s_{31} \partial s_{31}}\right) J_{2 \mid 4}=\frac{S_{1122} S_{2222}-S_{1222}^{2}}{4 I_{2}^{9 / 4}} \cdot\left(\left(144 z^{2}-24 z\right) \frac{\partial^{2} G(z)}{\partial z^{2}}+(216 z-12) \frac{\partial G(z)}{\partial z}+5 G(z)\right) \\
\left(\frac{\partial^{2}}{\partial s_{40} \partial s_{13}}-\frac{\partial^{2}}{\partial s_{31} \partial s_{22}}\right) J_{2 \mid 4}=\frac{S_{1222} S_{1122}-S_{1112} S_{2222}}{4 I_{2}^{9 / 4}} \cdot\left(\left(144 z^{2}-24 z\right) \frac{\partial^{2} G(z)}{\partial z^{2}}+(216 z-12) \frac{\partial G(z)}{\partial z}+5 G(z)\right) \\
\left(\frac{\partial^{2}}{\partial s_{40} \partial s_{04}}-\frac{\partial^{2}}{\partial s_{31} \partial s_{13}}\right) J_{2 \mid 4}=\frac{3 S_{1112} S_{1222}-3 S_{1122}^{2}}{4 I_{2}^{9 / 4}} \cdot\left(\left(144 z^{2}-24 z\right) \frac{\partial^{2} G(z)}{\partial z^{2}}+(216 z-12) \frac{\partial G(z)}{\partial z}+5 G(z)\right) \\
\left(\frac{\partial^{2}}{\partial s_{22} \partial s_{22}}-\frac{\partial^{2}}{\partial s_{31} \partial s_{13}}\right) J_{2 \mid 4}=\frac{S_{1111} S_{2222}-S_{1112} S_{1222}}{12 I_{2}^{9 / 4}} \cdot\left(\left(144 z^{2}-24 z\right) \frac{\partial^{2} G(z)}{\partial z^{2}}+(216 z-12) \frac{\partial G(z)}{\partial z}+5 G(z)\right)
\end{gathered}
$$

where $z=I_{3}^{2} / I_{2}^{3}$. These equations contain a common $S L(2)$ invariant factor. We conclude, that all the four expressions vanish, if and only if $G(z)$ satisfies the differential equation

$$
\left(144 z^{2}-24 z\right) \frac{\partial^{2} G(z)}{\partial z^{2}}+(216 z-12) \frac{\partial G(z)}{\partial z}+5 G(z)=0
$$

which is nothing but Gauss hypergeometric equation

$$
t(1-t) \frac{\partial^{2} G(t)}{\partial t^{2}}+(c-(a+b+1) t) \frac{\partial G(t)}{\partial t}-a b G(t)=0
$$

with $a=1 / 12, b=5 / 12, c=1 / 2$ and $t=6 z$. This is the invariant form of $n=2, r=4$ Ward identities.

Solution. In terms of the Gauss hypergeometric function

$$
{ }_{2} F_{1}([a, b],[c], t)=\sum_{k=0}^{\infty} \frac{\Gamma(a+k)}{\Gamma(a)} \frac{\Gamma(b+k)}{\Gamma(b)} \frac{\Gamma(c)}{\Gamma(c+k)} \frac{t^{k}}{k !}=1+\frac{a b}{c} t+\frac{a(a+1) b(b+1)}{c(c+1)} \frac{t^{2}}{2}+\ldots
$$

the general solution of Gauss hypergeometric equation is given by

$$
G(t)=c_{1} \cdot{ }_{2} F_{1}([a, b],[c], t)+c_{2} \cdot t^{1-c}{ }_{2} F_{1}([a+1-c, b+1-c],[2-c], t)
$$

Consequently, the integral discriminant equals

$$
J_{2 \mid 4}(S)=c_{1} \cdot I_{2}^{-1 / 4}{ }_{2} F_{1}\left(\left[\frac{1}{12}, \frac{5}{12}\right],\left[\frac{1}{2}\right], \frac{6 I_{3}^{2}}{I_{2}^{3}}\right)+c_{2} \cdot I_{3} I_{2}^{-7 / 4}{ }_{2} F_{1}\left(\left[\frac{7}{12}, \frac{11}{12}\right],\left[\frac{3}{2}\right], \frac{6 I_{3}^{2}}{I_{2}^{3}}\right)
$$

where $c_{1,2}$ are the two constants, parametrising the general solution of Ward identities. Particular solutions

$$
J_{2 \mid 4}^{(1)}(S)=I_{2}^{-1 / 4}{ }_{2} F_{1}\left(\left[\frac{1}{12}, \frac{5}{12}\right],\left[\frac{1}{2}\right], \frac{6 I_{3}^{2}}{I_{2}^{3}}\right) \quad \text { and } \quad J_{2 \mid 4}^{(2)}(S)=I_{3} I_{2}^{-7 / 4}{ }_{2} F_{1}\left(\left[\frac{7}{12}, \frac{11}{12}\right],\left[\frac{3}{2}\right], \frac{6 I_{3}^{2}}{I_{2}^{3}}\right)
$$

are associated with different integration contours and can be called the first and the second branches of $J_{2 \mid 4}$. 
Singularities. Notice, that the point $t=1$ corresponds to

$$
\frac{6 I_{3}^{2}}{I_{2}^{3}}=1
$$

which is just the discriminant's vanishing locus $I_{2}^{3}-6 I_{3}^{2}=D_{2 \mid 4}=0$. This is interesting, because the point $t=1$ is a singular point of the hypergeometric function ${ }_{2} F_{1}$. However, there are two other suspicious points: hypergeometric function ${ }_{2} F_{1}$ can have singularities at $t=0,1$ and $\infty$. Let us study asymptotics of integral discriminants at these points, using the formulas

$$
\begin{gathered}
{ }_{2} F_{1}([a, b],[c], t)=1+O(t), \quad \text { when } t \rightarrow 0 \\
{ }_{2} F_{1}([a, b],[a+b], 1-t)=-\frac{\Gamma(a+b)}{\Gamma(a) \Gamma(b)} \log t+O\left(t^{0}\right), \quad \text { when } t \rightarrow 0 \\
{ }_{2} F_{1}([a, b],[c], t)=t^{-a} \frac{\Gamma(b-a) \Gamma(c)}{\Gamma(b) \Gamma(c-a)} \cdot{ }_{2} F_{1}([a, a-c+1],[a-b+1], 1 / t)+ \\
t^{-b} \frac{\Gamma(a-b) \Gamma(c)}{\Gamma(a) \Gamma(c-b)} \cdot{ }_{2} F_{1}([b, b-c+1],[b-a+1], 1 / t)
\end{gathered}
$$

which can be found in any reference book of hypergeometric functions (see, e.g. [12]). Using (19), we find

$$
\begin{array}{cc}
J_{2 \mid 4}^{(1)}(S) \sim I_{2}^{-1 / 4}+O(t), \quad \text { when } t \rightarrow 0 \\
J_{2 \mid 4}^{(2)}(S) \sim I_{3} I_{2}^{-7 / 4}+O(t), \quad \text { when } t \rightarrow 0
\end{array}
$$

Using (20), we find

$$
\begin{array}{cc}
J_{2 \mid 4}^{(1)}(S) \sim \frac{-\Gamma(1 / 2)}{\Gamma(1 / 12) \Gamma(5 / 12)} I_{2}^{-1 / 4} \log \left(1-\frac{6 I_{3}^{2}}{I_{2}^{3}}\right)+O\left(t^{0}\right), \quad \text { when } t \rightarrow 1 \\
J_{2 \mid 4}^{(2)}(S) \sim \frac{-\Gamma(3 / 2)}{\Gamma(7 / 12) \Gamma(11 / 12)} I_{3} I_{2}^{-7 / 4} \log \left(1-\frac{6 I_{3}^{2}}{I_{2}^{3}}\right)+O\left(t^{0}\right), \quad \text { when } t \rightarrow 1
\end{array}
$$

Using (19) and (21), we find

$$
\begin{array}{ll}
J_{2 \mid 4}^{(1)}(S) \sim \frac{\Gamma(1 / 2) \Gamma(1 / 3)}{\Gamma(5 / 12)^{2}}(-6)^{-1 / 12} I_{3}^{-1 / 6}+O\left(t^{-5 / 12}\right), & \text { when } t \rightarrow \infty \\
J_{2 \mid 4}^{(2)}(S) \sim \frac{\Gamma(1 / 3) \Gamma(3 / 2)}{\Gamma(11 / 12)^{2}}(-6)^{-7 / 12} I_{3}^{-1 / 6}+O\left(t^{-11 / 12}\right), & \text { when } t \rightarrow \infty
\end{array}
$$

We can see from these results, that singularities of the integral discriminant $J_{2 \mid 4}$ at finite values of $I_{2}, I_{3}$ are completely controlled by the algebraic discriminant $D_{2 \mid 4}$. The singularity at discriminant locus is logarithmic. Other singularities, not related to the algebraic discriminant, are situated at infinite values of invariants: $I_{2} \rightarrow \infty(t \rightarrow 0)$ and $I_{3} \rightarrow \infty(t \rightarrow \infty)$. 
Hypergeometric integral. Function ${ }_{2} F_{1}$ has an integral representation

$$
{ }_{2} F_{1}([a, b],[c], t)=\frac{\Gamma(c)}{\Gamma(b) \Gamma(c-b)} \cdot \int_{0}^{1} d s \frac{s^{b-1}(1-s)^{c-b-1}}{(1-s t)^{a}}
$$

which is a direct consequence of a simpler identity

$$
\frac{\Gamma(a) \Gamma(b)}{\Gamma(a+b)}=\int_{0}^{1} d s s^{a-1}(1-s)^{b-1}
$$

For the integral discriminant, we obtain

$$
J_{2 \mid 4}(S)=c_{1} \cdot \int_{0}^{1} d s \frac{s^{-7 / 12}(1-s)^{-11 / 12}}{\left(I_{2}^{3}-6 s I_{3}^{2}\right)^{1 / 12}}+c_{2} I_{3} \cdot \int_{0}^{1} d s \frac{s^{-1 / 12}(1-s)^{-5 / 12}}{\left(I_{2}^{3}-6 s I_{3}^{2}\right)^{7 / 12}}
$$

Hypergeometric functions with different values of parameters $a, b$ and $c$ are related by various "modular" transformations of the variable $t$ and the integration variable $s$, which leave the boundary region (two points 0 and 1) intact. Such transformations were first found and studied by Euler, therefore they are known as Euler hypergeometric transformations. Say, transformation $t \mapsto t, s \mapsto 1-s$ gives rise to a relation

$$
{ }_{2} F_{1}([a, b],[c], t)=(1-t)^{-a} \cdot{ }_{2} F_{1}\left([a, c-b],[c], \frac{t}{t-1}\right)
$$

After this transformation, $J_{2 \mid 4}$ takes form

$$
\begin{gathered}
J_{2 \mid 4}^{(1)}(S)=\left(D_{2 \mid 4}\right)^{-1 / 12}{ }_{2} F_{1}\left(\left[\frac{1}{12}, \frac{1}{12}\right],\left[\frac{1}{2}\right],-\frac{6 I_{3}^{2}}{D_{2 \mid 4}}\right) \\
J_{2 \mid 4}^{(2)}(S)=I_{3}\left(D_{2 \mid 4}\right)^{-7 / 12}{ }_{2} F_{1}\left(\left[\frac{7}{12}, \frac{7}{12}\right],\left[\frac{3}{2}\right],-\frac{6 I_{3}^{2}}{D_{2 \mid 4}}\right)
\end{gathered}
$$

In this way the hypergeometric integral (24) allows to recast $J_{2 \mid 4}$ in various forms and establish relations between them. Therefore, (24) is a useful and important representation. We emphasise, that the relation of integral $\int e^{-S(x, y)} d x d y$ to hypergeometric integrals (24) is not a priori expected: these integrals look very different, even from the point of view of variables they depend on.

Vertical symmetry. To finish this section, let us check the vertical symmetry between $J_{2 \mid 2}$ and $J_{2 \mid 4}$ :

$$
J_{2 \mid 4}\left(\left(a x^{2}+b x y+c y^{2}\right)^{2}\right) \sim J_{2 \mid 2}\left(a x^{2}+b x y+c y^{2}\right)
$$

Setting $S(x, y)=\left(a x^{2}+b x y+c y^{2}\right)^{2}$ we obtain the coefficients

$$
S_{1111}=a^{2}, \quad S_{1112}=\frac{1}{2} a b, \quad S_{1122}=\frac{1}{3} a c+\frac{1}{6} b^{2}, \quad S_{1222}=\frac{1}{2} b c, \quad S_{2222}=c^{2}
$$


and the invariants

$$
I_{2}=\frac{1}{6}\left(b^{2}-4 a c\right)^{2}, \quad I_{3}=\frac{1}{36}\left(b^{2}-4 a c\right)^{3}
$$

Substituting them into (18), we find

$$
\begin{aligned}
& J_{2 \mid 4}^{(1)}(S)=\frac{A^{(1)}}{\sqrt{b^{2}-4 a c}} \\
& J_{2 \mid 4}^{(2)}(S)=\frac{A^{(2)}}{\sqrt{b^{2}-4 a c}}
\end{aligned}
$$

where the constants of proportionality

$$
\begin{aligned}
& A^{(1)}=6^{-1 / 4} \cdot{ }_{2} F_{1}\left(\left[\frac{1}{12}, \frac{5}{12}\right],\left[\frac{1}{2}\right], 1\right) \sim-6^{-1 / 4} \frac{\Gamma(1 / 2)}{\Gamma(1 / 12) \Gamma(5 / 12)} \log 0 \sim \infty \\
& A^{(2)}=6^{+1 / 4} \cdot{ }_{2} F_{1}\left(\left[\frac{7}{12}, \frac{11}{12}\right],\left[\frac{3}{2}\right], 1\right) \sim-6^{+1 / 4} \frac{\Gamma(3 / 2)}{\Gamma(7 / 12) \Gamma(11 / 12)} \log 0 \sim \infty
\end{aligned}
$$

are independent of $a, b, c$ - as prescribed by the vertical symmetry - but infinite. Only their linear combination

$$
\left(6^{+1 / 4} \frac{\Gamma(3 / 2)}{\Gamma(7 / 12) \Gamma(11 / 12)}\right) A^{(1)}-\left(6^{-1 / 4} \frac{\Gamma(1 / 2)}{\Gamma(1 / 12) \Gamma(5 / 12)}\right) A^{(2)}=\text { finite number }=-\frac{1}{2}
$$

is finite - logarithmic divergencies cancel out. Actually, it is expectable that only one linear combination of solutions stays finite. This is because not every contour, admissible for $J_{2 \mid 4}$, is admissible for $J_{2 \mid 2}$. Admissible contours for $J_{2 \mid 4}$ approach infinity from 4 different directions, where the 4 -form $\left(a x^{2}+b x y+c y^{2}\right)^{2}$ takes real positive values. Only two of these directions are appropriate for $J_{2 \mid 2}$ - those, where the 2 -form $a x^{2}+b x y+c y^{2}$ takes real positive values. One linear combination of branches corresponds to admissible contours, while the orthogonal linear combination corresponds to other contours. That is why both branches are singular at $t \rightarrow 1$, but certain linear combination of branches is regular at $t=1$. Note, that nothing similar happens at non-discriminantal singularities: at $t \rightarrow 0$ and $t \rightarrow \infty$ no linear combination of branches stays regular.

\subsection{The case of $J_{2 \mid 5}$}

Our third example is a 5 -form in 2 variables, which can be written as

$$
S(x, y)=S_{11111} x^{5}+5 S_{11112} x^{4} y+10 S_{11122} x^{3} y^{2}+10 S_{11222} x^{2} y^{3}+5 S_{12222} x y^{4}+S_{22222} y^{5}
$$

Invariants. By dimension counting, there are three elementary invariants in this case. They have degrees 4, 8 and 12, denoted as $I_{4}, I_{8}$ and $I_{12}$ and given by diagrams at Fig. 8, Fig. 9 and Fig. 10. Looking at the diagrams, it is straightforward to write an expression for $I_{4}$

$$
I_{4}=S_{i_{1} i_{2} i_{3} i_{4} i_{5}} S_{j_{1} j_{2} j_{3} j_{4} j_{5}} S_{k_{1} k_{2} k_{3} k_{4} k_{5}} S_{l_{1} l_{2} l_{3} l_{4} l_{5}} \epsilon^{i_{1} j_{1}} \epsilon^{i_{2} j_{2}} \epsilon^{i_{3} j_{3}} \epsilon^{i_{4} k_{4}} \epsilon^{i_{5} k_{5}} \epsilon^{j_{4} l_{4}} \epsilon^{j_{5} l_{5}} \epsilon^{k_{1} l_{1}} \epsilon^{k_{2} l_{2}} \epsilon^{k_{3} l_{3}}
$$

and equally straightforward to write expressions for $I_{8}, I_{12}$. Evaluating the contraction, one gets a formula 


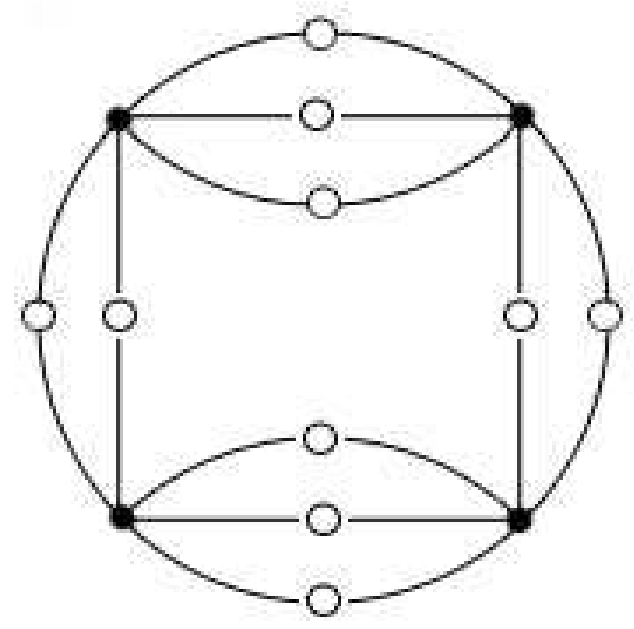

Figure 8: The degree 4 invariant $I_{4}$ of a 5 -form in 2 variables, represented as a diagram of tensor contraction. Black 5 -valent vertices represent tensor $S$, white 2-valent vertices represent tensor $\epsilon$.

$$
\begin{aligned}
I_{4}= & 2 S_{11111}^{2} S_{22222}^{2}-20 S_{11111} S_{11112} S_{12222} S_{22222}+8 S_{11111} S_{11122} S_{11222} S_{22222}+ \\
& 32 S_{11111} S_{11122} S_{12222}^{2}-24 S_{11111} S_{11222}^{2} S_{12222}+32 S_{11112}^{2} S_{11222} S_{22222}+18 S_{11112}^{2} S_{12222}^{2}- \\
& 24 S_{11112} S_{11122}^{2} S_{22222}-152 S_{11112} S_{11122} S_{11222} S_{12222}+96 S_{11112} S_{11222}^{3}+96 S_{11122}^{3} S_{12222}-64 S_{11122}^{2} S_{11222}^{2}
\end{aligned}
$$

and similar formulas for $I_{8}, I_{12}$ - they are quite lengthy and we do not present them here. The algebraic discriminant $D_{2 \mid 5}$, just like any other $S L(2)$-invariant function of $S$, is a function of $I_{4}, I_{8}, I_{12}$ :

$$
D_{2 \mid 5}=I_{4}^{2}-64 I_{8}
$$

Integral discriminant. Similarly, the integral discriminant is a function of $I_{4}, I_{8}, I_{12}$ :

$$
J_{2 \mid 5}=F\left(I_{4}, I_{8}, I_{12}\right)
$$

where the function $F$ is to be determined. The homogeneity condition does not allow to find $F$ unambigously, it constrains only the overall scaling factor, but not the non-trivial dependence on the ratios of invariants:

$$
F\left(I_{4}, I_{8}, I_{12}\right)=I_{4}^{-1 / 10} G\left(\frac{I_{8}}{I_{4}^{2}}, \frac{I_{12}}{I_{4}^{3}}\right)
$$

To find the function $G(u, v)$ in this ansatz, we need to use the Ward identities (8). 


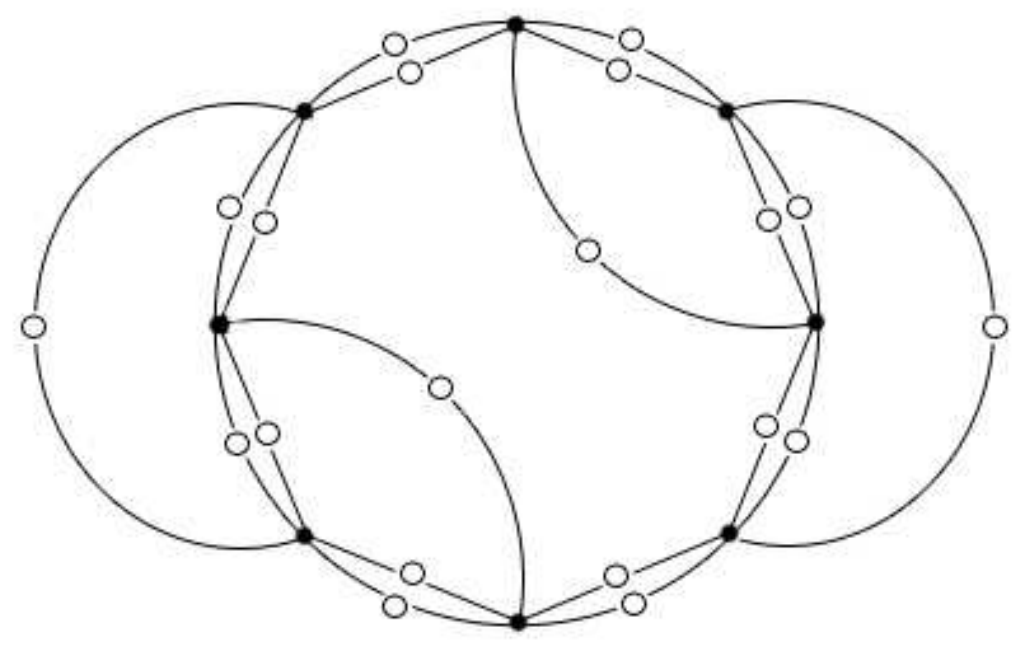

Figure 9: The degree 8 invariant $I_{8}$ of a 5 -form in 2 variables, represented as a diagram of tensor contraction. Black 5-valent vertices represent tensor $S$, white 2-valent vertices represent tensor $\epsilon$.

Ward identities. Applied to the present case of $n=2, r=5$, the system (8) takes the form

$$
\begin{aligned}
& \left(\frac{\partial^{2}}{\partial s_{50} \partial s_{32}}-\frac{\partial^{2}}{\partial s_{41} \partial s_{41}}\right) J_{2 \mid 5}=0 \quad\left(\frac{\partial^{2}}{\partial s_{32} \partial s_{32}}-\frac{\partial^{2}}{\partial s_{41} \partial s_{23}}\right) J_{2 \mid 5}=0 \\
& \left(\frac{\partial^{2}}{\partial s_{50} \partial s_{23}}-\frac{\partial^{2}}{\partial s_{41} \partial s_{32}}\right) J_{2 \mid 5}=0 \quad\left(\frac{\partial^{2}}{\partial s_{50} \partial s_{05}}-\frac{\partial^{2}}{\partial s_{32} \partial s_{23}}\right) J_{2 \mid 5}=0 \\
& \left(\frac{\partial^{2}}{\partial s_{50} \partial s_{14}}-\frac{\partial^{2}}{\partial s_{41} \partial s_{23}}\right) J_{2 \mid 5}=0 \quad\left(\frac{\partial^{2}}{\partial s_{41} \partial s_{14}}-\frac{\partial^{2}}{\partial s_{32} \partial s_{23}}\right) J_{2 \mid 5}=0
\end{aligned}
$$

where $s$-parameters and $S$-parameters are related by

$$
s_{50}=S_{11111}, s_{41}=5 S_{11112}, s_{32}=10 S_{11122}, s_{23}=10 S_{11222}, s_{14}=5 S_{12222}, s_{05}=S_{22222}
$$

Particular equations in this system are, of course, not $S L(2)$ invariant.

Invariant form of the Ward identities. It is possible to deal with non-invariant equations (as we did in the previous section) but it is much more convenient to rewrite the above differential equations in $S L(2)$-invariant form, by summing them with appropriate $S$-dependent coefficients:

$$
\begin{aligned}
\hat{O}_{0}= & \left(2 S_{11111} S_{12222}-8 S_{11112} S_{11222}+6 S_{11122}^{2}\right)\left(2 \frac{\partial}{\partial s_{50}} \frac{\partial}{\partial s_{14}}-8 \frac{\partial}{\partial s_{41}} \frac{\partial}{\partial s_{23}}+6 \frac{\partial}{\partial s_{32}} \frac{\partial}{\partial s_{32}}\right)+ \\
& 2\left(S_{11111} S_{22222}-3 S_{11112} S_{12222}+2 S_{11122} S_{11222}\right)\left(\frac{\partial}{\partial s_{05}} \frac{\partial}{\partial s_{50}}-3 \frac{\partial}{\partial s_{41}} \frac{\partial}{\partial s_{14}}+2 \frac{\partial}{\partial s_{32}} \frac{\partial}{\partial s_{23}}\right)+ \\
& \left(2 S_{11112} S_{22222}-8 S_{11122} S_{12222}+6 S_{11222}^{2}\right)\left(2 \frac{\partial}{\partial s_{41}} \frac{\partial}{\partial s_{05}}-8 \frac{\partial}{\partial s_{32}} \frac{\partial}{\partial s_{14}}+6 \frac{\partial}{\partial s_{23}} \frac{\partial}{\partial s_{23}}\right)
\end{aligned}
$$




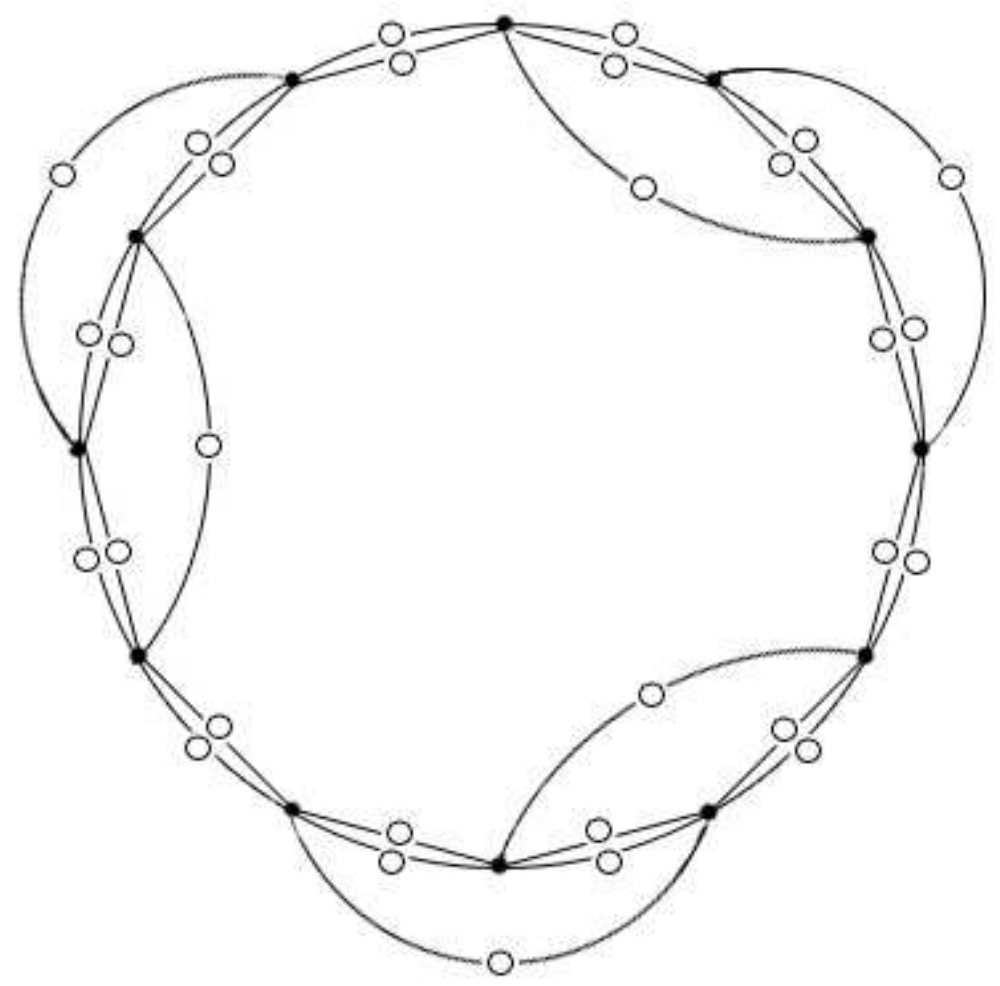

Figure 10: The degree 12 invariant $I_{12}$ of a 5 -form in 2 variables, represented as a diagram of tensor contraction. Black 5 -valent vertices represent tensor $S$, white 2 -valent vertices represent tensor $\epsilon$.

and

$$
\begin{aligned}
\hat{O}_{4}= & P_{11}(S)\left(2 \frac{\partial}{\partial s_{50}} \frac{\partial}{\partial s_{14}}-8 \frac{\partial}{\partial s_{41}} \frac{\partial}{\partial s_{23}}+6 \frac{\partial}{\partial s_{32}} \frac{\partial}{\partial s_{32}}\right)+ \\
& 2 P_{12}(S)\left(\frac{\partial}{\partial s_{05}} \frac{\partial}{\partial s_{50}}-3 \frac{\partial}{\partial s_{41}} \frac{\partial}{\partial s_{14}}+2 \frac{\partial}{\partial s_{32}} \frac{\partial}{\partial s_{23}}\right)+ \\
& P_{22}(S)\left(2 \frac{\partial}{\partial s_{41}} \frac{\partial}{\partial s_{05}}-8 \frac{\partial}{\partial s_{32}} \frac{\partial}{\partial s_{14}}+6 \frac{\partial}{\partial s_{23}} \frac{\partial}{\partial s_{23}}\right)
\end{aligned}
$$

where $P$ is a quadratic form with coefficients

$$
\begin{aligned}
P_{a b}(S)= & S_{a i_{2} i_{3} i_{4} i_{5}} S_{j_{1} j_{2} j_{3} j_{4} j_{5}} S_{k_{1} k_{2} k_{3} k_{4} k_{5}} S_{l_{1} l_{2} l_{3} l_{4} l_{5}} S_{m_{1} m_{2} m_{3} m_{4} m_{5}} S_{s_{1} s_{2} s_{3} s_{4} b} \\
& \epsilon^{i_{2} j_{2}} \epsilon^{i_{3} j_{3}} \epsilon^{i_{4} k_{4}} \epsilon^{i_{5} k_{5}} \epsilon^{k_{1} l_{1}} \epsilon^{k_{2} l_{2}} \epsilon^{j_{4} m_{4}} \epsilon^{j_{5} m_{5}} \epsilon^{m_{1} s_{1}} \epsilon^{m_{2} s_{2}} \epsilon^{l_{3} s_{3}} \epsilon^{l_{4} s_{4}} \epsilon^{j_{1} k_{3}} \epsilon^{l_{5} m_{3}}
\end{aligned}
$$

Operators $\hat{O}_{0}$ and $\hat{O}_{4}$ are $S L(2)$-invariant, simply because they are given by diagrams at Fig.11 and Fig.12. The subscripts " 0 " and " 4 " stand for the degrees of these operators. In analogy with invariants $I_{k}$, we denote the invariant differential operators of degree $k$ as $\hat{O}_{k}$. 


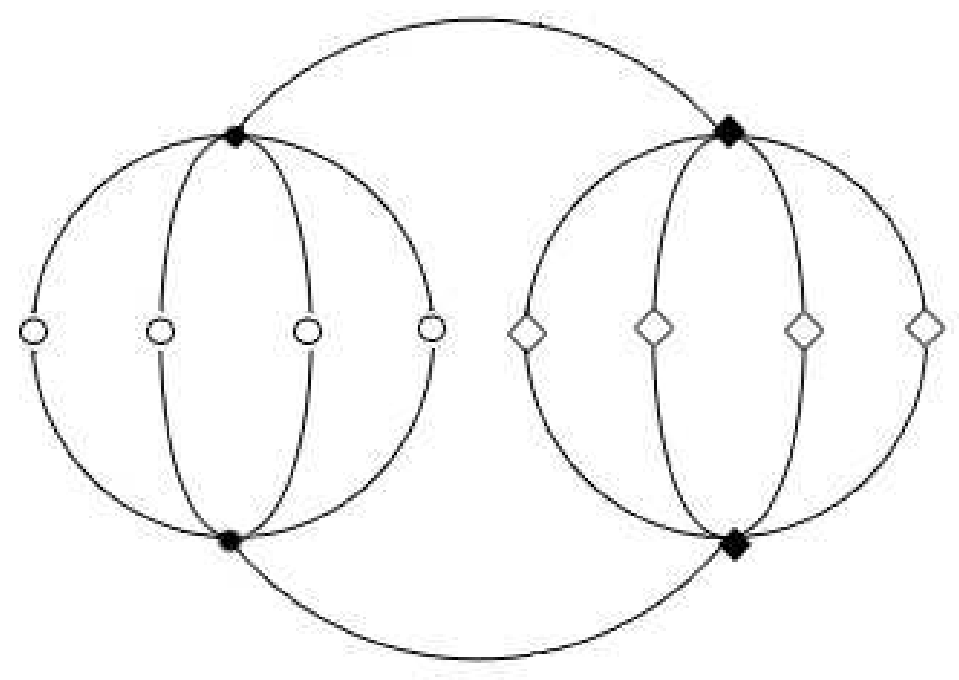

Figure 11: Invariant differential operator $\hat{O}_{0}$ for the case $2 \mid 5$, represented as a diagram of tensor contraction. Black 5 -valent circles represent tensor $S$, white 2 -valent circles represent tensor $\epsilon$, black 5 -valent rhombuses represent tensor $\partial / \partial S$, white 2 -valent rhombuses represent tensor $\epsilon_{*}$.

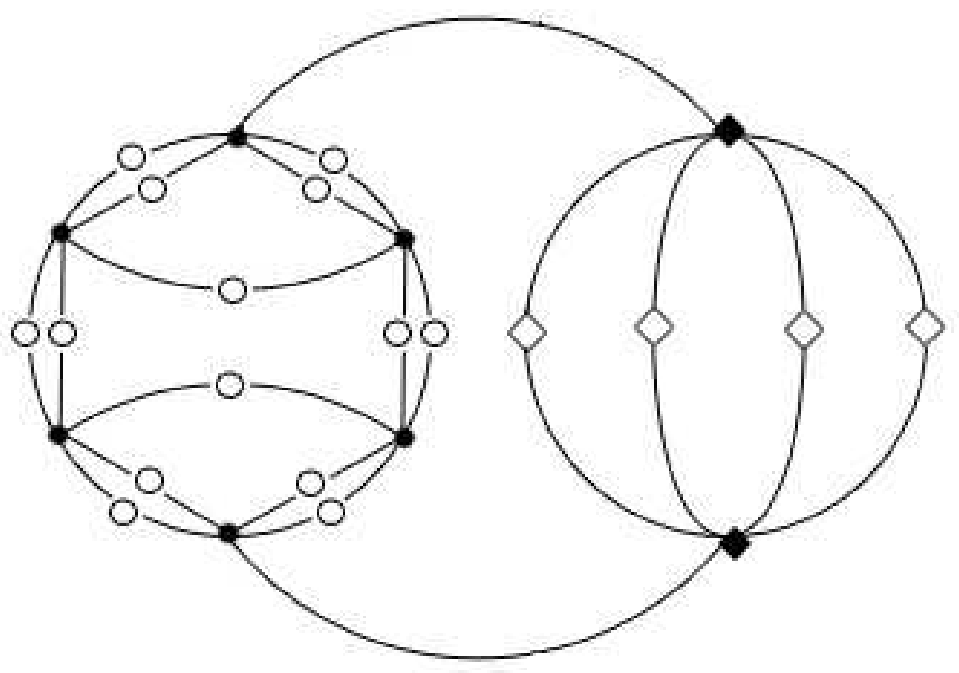

Figure 12: Invariant differential operator $\hat{O}_{4}$ for the case $2 \mid 5$, represented as a diagram of tensor contraction. Black 5 -valent circles represent tensor $S$, white 2 -valent circles represent tensor $\epsilon$, black 5 -valent rhombuses represent tensor $\partial / \partial S$, white 2-valent rhombuses represent tensor $\epsilon_{*}$. 
Of course, operators $\hat{O}_{0}$ and $\hat{O}_{4}$ are not unique - there are many other invariant differential operators, which annihilate $J_{2 \mid 5}$. All such operators contain an $\epsilon$-antisymmetrized combination of two derivatives $\partial / \partial S$, which is exactly the structure of Ward identities. We do not study here the interesting problems of classification of these operators and finding relations between them, because $\hat{O}_{0}$ and $\hat{O}_{4}$ are quite enough to find $J_{2 \mid 5}$. Let us derive a useful formula for the action of second order operators

$$
\hat{O}=\sum_{\alpha, \beta} C_{\alpha, \beta} \frac{\partial}{\partial S_{\alpha}} \frac{\partial}{\partial S_{\beta}}
$$

By application of the chain rule, we obtain

$$
\hat{O} F\left\{I_{k}\right\}=\sum_{k} \frac{\partial F}{\partial I_{k}} \sum_{\alpha, \beta} C_{\alpha, \beta} \frac{\partial^{2} I_{k}}{\partial S_{\alpha} \partial S_{\beta}}+\sum_{k, m} \frac{\partial^{2} F}{\partial I_{k} \partial I_{m}} \sum_{\alpha, \beta} C_{\alpha, \beta} \frac{\partial I_{k}}{\partial S_{\alpha}} \frac{\partial I_{m}}{\partial S_{\beta}}
$$

Since

$$
\sum_{\alpha, \beta} C_{\alpha, \beta} \frac{\partial^{2} I_{k}}{\partial S_{\alpha} \partial S_{\beta}}=\hat{O} I_{k}
$$

and

$$
\sum_{\alpha, \beta} 2 C_{\alpha, \beta} \frac{\partial I_{k}}{\partial S_{\alpha}} \frac{\partial I_{m}}{\partial S_{\beta}}=\hat{O}\left(I_{k} I_{m}\right)-I_{k} \hat{O} I_{m}-I_{m} \hat{O} I_{k}
$$

we finally obtain an important formula:

$$
\hat{O} F\left\{I_{k}\right\}=\sum_{k} \frac{\partial F}{\partial I_{k}} \hat{O} I_{k}+\frac{1}{2} \sum_{k, m} \frac{\partial^{2} F}{\partial I_{k} \partial I_{m}}\left[\hat{O}\left(I_{k} I_{m}\right)-I_{k} \hat{O} I_{m}-I_{m} \hat{O} I_{k}\right]
$$

As one can see, to describe the action of $\hat{O}$-operators on arbitrary invariant functions it suffices to calculate the action on all invariants $I_{k}$ and all products $I_{k} I_{m}$. In the present case we have

$$
\begin{aligned}
& \hat{O}_{0}\left(\begin{array}{c}
I_{4} \\
I_{8} \\
I_{12}
\end{array}\right)=\left(\begin{array}{c}
\frac{264}{25} I_{4} \\
\frac{2}{25} I_{4}^{2}+\frac{294}{25} I_{8} \\
\frac{12}{25} I_{4} I_{8}+\frac{162}{5} I_{12}
\end{array}\right) \\
& \hat{O}_{0}\left(\begin{array}{ccc}
I_{4}^{2} & I_{4} I_{8} & I_{4} I_{12} \\
I_{4} I_{8} & I_{8}^{2} & I_{8} I_{12} \\
I_{4} I_{12} & I_{8} I_{12} & I_{12}^{2}
\end{array}\right)=\left(\begin{array}{ccc}
\frac{928}{25} I_{4}^{2}-\frac{384}{25} I_{8} & \frac{2}{25} I_{4}^{3}+\frac{1166}{25} I_{4} I_{8}+\frac{192}{5} I_{12} & \frac{12}{25} I_{4}^{2} I_{8}+\frac{144}{25} I_{8}^{2}+\frac{1794}{25} I_{4} I_{12} \\
\frac{2}{25} I_{4}^{3}+\frac{1166}{25} I_{4} I_{8}+\frac{192}{5} I_{12} & \frac{8}{25} I_{4}^{2} I_{8}+\frac{1188}{25} I_{8}^{2}+\frac{12}{5} I_{4} I_{12} & \frac{9}{25} I_{4} I_{8}^{2}+\frac{12}{25} I_{4}^{2} I_{12}+\frac{1944}{25} I_{8} I_{12} \\
\frac{12}{25} I_{4}^{2} I_{8}+\frac{144}{25} I_{8}^{2}+\frac{1794}{25} I_{4} I_{12} & \frac{9}{25} I_{4} I_{8}^{2}+\frac{12}{25} I_{4}^{2} I_{12}+\frac{1944}{25} I_{8} I_{12} & -\frac{54}{25} I_{8}^{3}+\frac{84}{25} I_{4} I_{8} I_{12}+\frac{684}{5} I_{12}^{2}
\end{array}\right)
\end{aligned}
$$


and similarly for the second operator

$$
\hat{O}_{4}\left(\begin{array}{c}
I_{4} \\
I_{8} \\
I_{12}
\end{array}\right)=\left(\begin{array}{c}
-\frac{264}{25} I_{8} \\
-\frac{2}{25} I_{4} I_{8}+\frac{588}{25} I_{12} \\
\frac{363}{50} I_{8}^{2}-\frac{153}{25} I_{4} I_{12}
\end{array}\right)
$$

$\hat{O}_{4}\left(\begin{array}{ccc}I_{4}^{2} & I_{4} I_{8} & I_{4} I_{12} \\ I_{4} I_{8} & I_{8}^{2} & I_{8} I_{12} \\ I_{4} I_{12} & I_{8} I_{12} & I_{12}^{2}\end{array}\right)=\left(\begin{array}{ccc}-\frac{928}{25} I_{4} I_{8}-\frac{768}{25} I_{12} & -\frac{2}{25} I_{4}^{2} I_{8}-\frac{584}{25} I_{8}^{2}+\frac{524}{25} I_{4} I_{12} & \frac{363}{50} I_{4} I_{8}^{2}-\frac{153}{25} I_{4}^{2} I_{12}-\frac{696}{25} I_{8} I_{12} \\ -\frac{2}{25} I_{4}^{2} I_{8}-\frac{584}{25} I_{8}^{2}+\frac{524}{25} I_{4} I_{12} & \frac{1}{25} I_{4} I_{8}^{2}-\frac{22}{25} I_{4}^{2} I_{12}+\frac{2376}{25} I_{8} I_{12} & \frac{603}{50} I_{8}^{3}-\frac{291}{25} I_{4} I_{8} I_{12}+\frac{1188}{25} I_{12}^{2} \\ \frac{363}{50} I_{4} I_{8}^{2}-\frac{153}{25} I_{4}^{2} I_{12}-\frac{696}{25} I_{8} I_{12} & \frac{603}{50} I_{8}^{3}-\frac{291}{25} I_{4} I_{8} I_{12}+\frac{1188}{25} I_{12}^{2} & \frac{129}{5} I_{8}^{2} I_{12}-\frac{606}{25} I_{4} I_{12}^{2}\end{array}\right)$

Applying (28), we obtain

$$
\begin{gathered}
\hat{O}_{0} F\left(I_{4}, I_{8}, I_{12}\right)=\frac{264}{25} I_{4} \frac{\partial F}{\partial I_{4}}+\left(\frac{2}{25} I_{4}^{2}+\frac{294}{25} I_{8}\right) \frac{\partial F}{\partial I_{8}}+\left(\frac{12}{25} I_{4} I_{8}+\frac{162}{5} I_{12}\right) \frac{\partial F}{\partial I_{12}} \\
+\left(8 I_{4}^{2}-\frac{192}{25} I_{8}\right) \frac{\partial^{2} F}{\partial I_{4}^{2}}+\left(\frac{2}{25} I_{4}^{2} I_{8}+12 I_{8}^{2}+\frac{6}{5} I_{4} I_{12}\right) \frac{\partial^{2} F}{\partial I_{8}^{2}}+\left(-\frac{27}{25} I_{8}^{3}+\frac{6}{5} I_{4} I_{8} I_{12}+36 I_{12}^{2}\right) \frac{\partial^{2} F}{\partial I_{12}^{2}} \\
+\left(\frac{608}{25} I_{4} I_{8}+\frac{192}{5} I_{12}\right) \frac{\partial^{2} F}{\partial I_{4} \partial I_{8}}+\left(\frac{144}{25} I_{8}^{2}+\frac{144}{5} I_{4} I_{12}\right) \frac{\partial^{2} F}{\partial I_{4} \partial I_{12}}+\left(-\frac{3}{25} I_{4} I_{8}^{2}+\frac{2}{5} I_{4}^{2} I_{12}+\frac{168}{5} I_{8} I_{12}\right) \frac{\partial^{2} F}{\partial I_{8} \partial I_{12}}
\end{gathered}
$$

and similarly for the second operator

$$
\begin{gathered}
\hat{O}_{4} F\left(I_{4}, I_{8}, I_{12}\right)=-\frac{264}{25} I_{8} \frac{\partial F}{\partial I_{4}}+\left(-\frac{2}{25} I_{4} I_{8}+\frac{588}{25} I_{12}\right) \frac{\partial F}{\partial I_{8}}+\left(\frac{363}{50} I_{8}^{2}-\frac{153}{25} I_{4} I_{12}\right) \frac{\partial F}{\partial I_{12}} \\
+\left(-8 I_{4} I_{8}-\frac{384}{25} I_{12}\right) \frac{\partial^{2} F}{\partial I_{4}^{2}}+\left(\frac{1}{10} I_{4} I_{8}^{2}-\frac{11}{25} I_{4}^{2} I_{12}+24 I_{8} I_{12}\right) \frac{\partial^{2} F}{\partial I_{8}^{2}}+\left(\frac{141}{25} I_{8}^{2} I_{12}-6 I_{4} I_{12}^{2}\right) \frac{\partial^{2} F}{\partial I_{12}^{2}} \\
+\left(-\frac{64}{5} I_{8}^{2}-\frac{64}{25} I_{4} I_{12}\right) \frac{\partial^{2} F}{\partial I_{4} \partial I_{8}}-\frac{432}{25} I_{8} I_{12} \frac{\partial^{2} F}{\partial I_{4} \partial I_{12}}+\left(\frac{24}{5} I_{8}^{3}-\frac{136}{25} I_{4} I_{8} I_{12}+24 I_{12}^{2}\right) \frac{\partial^{2} F}{\partial I_{8} \partial I_{12}}
\end{gathered}
$$

For the integral discriminant, both expressions vanish. Substituting the ansatz (27) and making the necessary algebraic transformations, we obtain two differential equations on the function $G(u, v)$ : the first

$$
\begin{aligned}
& 50(-1+64 u)\left(u+6 u^{2}+15 v\right) \frac{\partial^{2} G}{\partial u^{2}}+\left(75 u^{2}+72000 v^{2}+57600 v u^{2}+600 v u+7200 u^{3}-250 v\right) \frac{\partial^{2} G}{\partial u \partial v}+ \\
& \left(675 u^{3}-13500 v^{2}+10800 v u^{2}-750 v u+43200 u v^{2}\right) \frac{\partial^{2} G}{\partial v^{2}}+\left(50400 v+30720 u^{2}-50+5770 u\right) \frac{\partial G}{\partial u}+ \\
& \left(-300 u+60480 v u+11160 u^{2}-7650 v\right) \frac{\partial G}{\partial v}+(528 u+110) G=0
\end{aligned}
$$


and the second

$$
\begin{aligned}
& 25(-1+64 u)\left(5 u^{2}+48 u v-22 v\right) \frac{\partial^{2} G}{\partial u^{2}}+\left(230400 u v^{2}-39600 v^{2}-6000 u^{3}+28800 v u^{2}+6800 v u\right) \frac{\partial^{2} G}{\partial u \partial v}+ \\
& \left(172800 v^{3}+7500 v^{2}+25200 u v^{2}-7050 v u^{2}\right) \frac{\partial^{2} G}{\partial v^{2}}+\left(-36120 v+4000 u^{2}+122880 v u+100 u\right) \frac{\partial G}{\partial u}+ \\
& \left(241920 v^{2}+19440 v u-9075 u^{2}+7650 v\right) \frac{\partial G}{\partial v}+(-220 u+2112 v) G(u, v)=0
\end{aligned}
$$

where $u=I_{8} / I_{4}^{2}$ and $v=I_{12} / I_{4}^{3}$. These two linear differential equations in two variables constitute the invariant form of $n=2, r=5$ Ward identities. Integral discriminant is found as the solution of this system.

Solution. Having linear differential equations, it is easy to solve them in series: if one puts

$$
G(u, v)=\sum_{i, j} c_{i j} u^{i} v^{j}
$$

then one finds, after some algebraic transformations,

$$
c_{i j}=\mathrm{const} \cdot \frac{1}{i ! j !} \cdot 16^{i}\left(\frac{128}{3}\right)^{j} \cdot \frac{\Gamma\left(\frac{3}{10}+i+j\right) \Gamma\left(\frac{1}{10}+2 i+3 j\right) \Gamma\left(\frac{1}{10}+j\right)}{\Gamma\left(\frac{2}{5}+i+2 j\right) \Gamma\left(\frac{3}{5}+i+2 j\right)}
$$

i.e. there is unique series solution. In this way one obtains the first branch of the integral discriminant $J_{2 \mid 5}$ :

$$
J_{2 \mid 5}^{(1)}(S)=I_{4}^{-1 / 10} \sum_{i, j} \frac{\Gamma\left(\frac{3}{10}+i+j\right) \Gamma\left(\frac{1}{10}+2 i+3 j\right) \Gamma\left(\frac{1}{10}+j\right)}{\Gamma\left(\frac{2}{5}+i+2 j\right) \Gamma\left(\frac{3}{5}+i+2 j\right)} \cdot \frac{1}{i ! j !} \cdot\left(\frac{16 I_{8}}{I_{4}^{2}}\right)^{i}\left(\frac{128 I_{12}}{3 I_{4}^{3}}\right)^{j}
$$

This answer is interesting: the function appears to be of generalised hypergeometric type [9], since its coefficients $c_{i j}$ are ratios of $\Gamma$-functions, depending on linear combinations of indices $i$ and $j$. However, these series have a rather small convergence radius. Already the subsequence with $i=j$ behaves as

$$
\sum_{j} \frac{\Gamma\left(\frac{3}{10}+2 j\right) \Gamma\left(\frac{1}{10}+5 j\right) \Gamma\left(\frac{1}{10}+j\right)}{\Gamma\left(\frac{2}{5}+3 j\right) \Gamma\left(\frac{3}{5}+3 j\right)}\left(\frac{2^{11}}{3} u v\right)^{j} \sim \sum_{j} \frac{1}{j^{2}}\left(\frac{2^{13} 5^{5}}{3^{7}} u v\right)^{j}
$$

and diverges when the combination in the last brackets exceeds unity. For analytical continuation, one better substitutes this series by its integral representation.

Hypergeometric integral. Applying the formula (23) twice, we get

$$
\frac{\Gamma\left(\frac{3}{10}+i+j\right) \Gamma\left(\frac{1}{10}+2 i+3 j\right) \Gamma\left(\frac{1}{10}+j\right)}{\Gamma\left(\frac{2}{5}+i+2 j\right) \Gamma\left(\frac{3}{5}+i+2 j\right)}=\frac{1}{\Gamma\left(\frac{1}{2}-i-j\right)} \int_{0}^{1} \int_{0}^{1} d t d s \frac{t^{-7 / 10}(s-t s)^{-9 / 10}}{\sqrt{1-s}}\left(\frac{t s^{2}}{1-s}\right)^{i}\left(\frac{t(1-t) s^{3}}{1-s}\right)^{j}
$$


The sum over $i$ and $j$ is calculated, using

$$
\sum_{i, j} \frac{1}{\Gamma\left(\frac{1}{2}-i-j\right)} \frac{A^{i}}{i !} \frac{B^{j}}{j !}=\frac{1}{\sqrt{1+A+B}}
$$

and we obtain the following integral representation:

$$
G(u, v)=\int_{0}^{1} \int_{0}^{1} d t d s \cdot \frac{t^{-7 / 10} s^{-9 / 10}(1-t)^{-9 / 10}}{\sqrt{3-3 s+48 u t s^{2}+128 v s^{3} t-128 v s^{3} t^{2}}}
$$

Accordingly, for $J_{2 \mid 5}$ we have

$$
J_{2 \mid 5}^{(1)}(S)=I_{4}^{7 / 5} \int_{0}^{1} \int_{0}^{1} d t d s \cdot \frac{t^{-7 / 10} s^{-9 / 10}(1-t)^{-9 / 10}}{\sqrt{3 I_{4}^{3}-3 I_{4}^{3} s+48 I_{4} I_{8} t s^{2}+128 I_{12} s^{3} t-128 I_{12} s^{3} t^{2}}}
$$

Other branches of $J_{2 \mid 5}$ can be obtained by various transformations of variables $(u, v)$ and integration variables $(t, s)$, which leave the boundary region intact ("modular" transformations). We do not list them in this paper.

Singularities. One of the benefits of integral representation is the possibility to analyse the singularities. Singularities of the integral (30) come from zeroes of the polynomial

$$
P(s, t)=3-3 s+48 u t s^{2}+128 v s^{3} t-128 v s^{3} t^{2}
$$

which stands in the denominator. Ordinary zeroes (say, in $t$ ) are inessential, already because the singularity $d t / \sqrt{t-t_{0}}$ is integrable, and - more important - because the integration contour can be deformed and taken away from the singularity. This can not be done if the two roots coincide, i.e. if discriminant of $P(t)$ is vanishing. Then what matters is integration over $s$. If

$$
\operatorname{Disc}_{t}(P(s, t)) \sim\left(t_{1}(s)-t_{2}(s)\right)^{2}
$$

has a simple zero at $s=s_{0}$, then

$$
P(s, t) \sim\left(t-t_{0}\right)^{2}+\left(t_{1}-t_{2}\right)^{2} \sim\left(t-t_{0}\right)^{2}+\left(s-s_{0}\right)
$$

and the integration contour for $s$ can be taken away from the singularity. More serious is the case when discriminant has double zero, i.e. when repeated discriminant vanishes,

$$
\operatorname{Disc}_{s}\left(\operatorname{Disc}_{t}(P(s, t))\right)=0
$$

Then contour can not be deformed, neither in $t$ nor in $s$. However, in the vicinity of such point 


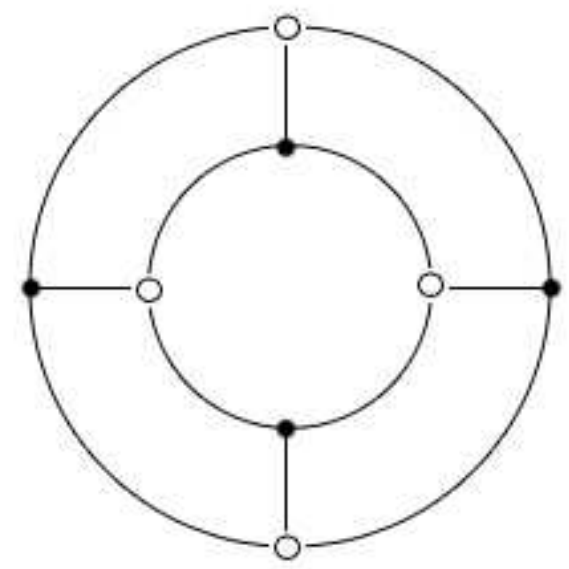

Figure 13: The degree 4 invariant $I_{4}$ of a 3 -form in 3 variables, represented as a diagram of tensor contraction. Black 3-valent vertices represent tensor $S$, white 3 -valent vertices represent tensor $\epsilon$.

$$
P(s, t) \sim\left(t-t_{0}\right)^{2}+\left(s-s_{0}\right)^{2}
$$

and this singularity, though unavoidable, is integrable because of the square root and because the integral is two-dimensional. This is most simply expressed in polar coordinates:

$$
\frac{d s d t}{\sqrt{\left(t-t_{0}\right)^{2}+\left(s-s_{0}\right)^{2}}} \sim d r d \phi
$$

Moreover, even if repeated discriminant has zeroes of higher order, $P(s, t) \sim\left(t-t_{0}\right)^{2}+\left(s-s_{0}\right)^{k}$, the singularity in the integral does not arise.

Thus the only remaining source of singularity is when it occurs at the ends of integration segments (at the boundary of integration domain - because the contour can not be deformed to avoid these boundaries), i.e. when $s$ or $t$ equals 0 or 1 . Given the shape of $P(s, t)$, of these the only significant one is at $t=1$, when $P(s, 1)=3-3 s+48 u s^{2}$. Remaining integral over $s$ becomes singular when this expression has a double zero:

$$
\operatorname{Disc}_{s}\left(3-3 s+48 u s^{2}\right)=1-64 u=0
$$

i.e. when $u=1 / 64$. This point corresponds to

$$
\frac{64 I_{8}}{I_{4}^{2}}=1
$$

which is just the discriminant's vanishing locus $I_{4}^{2}-64 I_{8}=D_{2 \mid 5}=0$. We encounter once again the same relation between integral and algebraic discriminants: the latter controls essential singularities of the former. Note, that the double zero occurs at the point $s=2$, which lies beyond the integration domain. For this reason, the singularity is rather soft. 


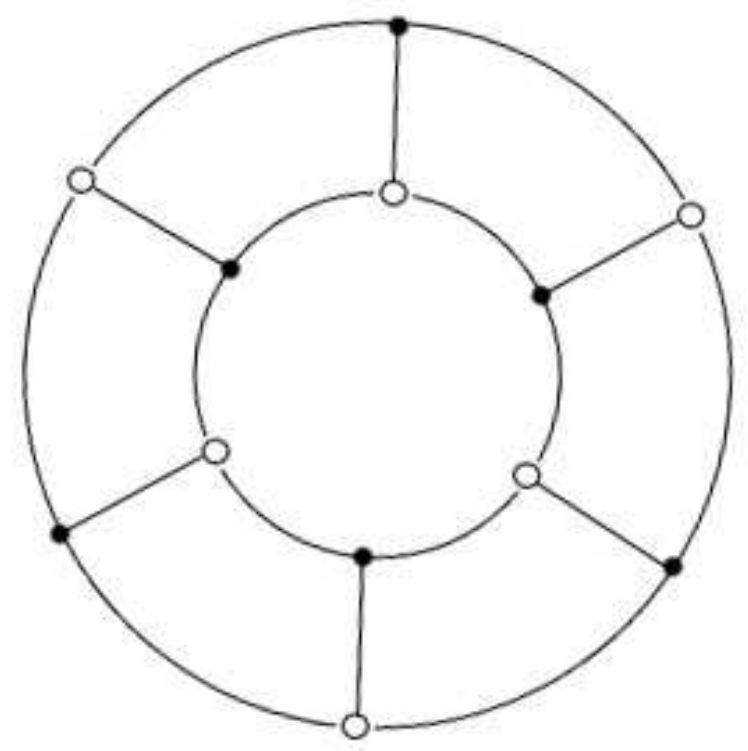

Figure 14: The degree 6 invariant $I_{6}$ of a 3 -form in 3 variables, represented as a diagram of tensor contraction. Black 3-valent vertices represent tensor $S$, white 3 -valent vertices represent tensor $\epsilon$.

\subsection{The case of $J_{3 \mid 3}$}

Our final example is a 3 -form in 3 variables, which can be written as

$S(x, y)=S_{111} x^{3}+3 S_{112} x^{2} y+3 S_{113} x^{2} z+S_{222} y^{3}+3 S_{122} x y^{2}+3 S_{223} y^{2} z+S_{333} z^{3}+3 S_{133} x z^{2}+3 S_{233} y z^{2}+6 S_{123} x y z$

Invariants. By dimension counting, there are two elementary invariants in this case. They have degrees 4 and 6 , denoted as $I_{4}, I_{6}$ and given by diagrams at Fig. 13, Fig. 14. Looking at the diagrams, it is straightforward to write the algebraic expressions for $I_{4}$ and $I_{6}$ :

$$
\begin{gathered}
I_{4}=S_{i_{1} i_{2} i_{3}} S_{j_{1} j_{2} j_{3}} S_{k_{1} k_{2} k_{3}} S_{l_{1} l_{2} l_{3}} \epsilon^{i_{1} j_{1} k_{1}} \epsilon^{i_{2} j_{2} l_{2}} \epsilon^{i_{3} k_{3} l_{3}} \epsilon^{l_{1} k_{2} j_{3}} \\
I_{6}=S_{i_{1} i_{2} i_{3}} S_{j_{1} j_{2} j_{3}} S_{k_{1} k_{2} k_{3}} S_{l_{1} l_{2} l_{3}} S_{m_{1} m_{2} m_{3}} S_{s_{1} s_{2} s_{3}} \epsilon^{i_{1} k_{1} l_{1}} \epsilon^{i_{2} j_{2} s_{2}} \epsilon^{j_{1} k_{2} m_{1}} \epsilon^{l_{2} m_{2} k_{3}} \epsilon^{m_{3} s_{3} j_{3}} \epsilon^{l_{3} i_{3} s_{1}}
\end{gathered}
$$

Evaluating these sums, one gets the following explicit formulas for $I_{4}, I_{6}$ :

$$
\begin{gathered}
I_{4}=6 S_{123}^{4}-12 S_{122} S_{123}^{2} S_{133}+6 S_{122}^{2} S_{133}^{2}+6 S_{113} S_{123} S_{133} S_{222}-12 S_{113} S_{123}^{2} S_{223}-6 S_{113} S_{122} S_{133} S_{223}+ \\
18 S_{113} S_{122} S_{123} S_{233}-6 S_{113} S_{122}^{2} S_{333}+6 S_{113}^{2} S_{223}^{2}-6 S_{113}^{2} S_{222} S_{233}-6 S_{112} S_{133}^{2} S_{222}+18 S_{112} S_{123} S_{133} S_{223}- \\
12 S_{112} S_{123}^{2} S_{233}-6 S_{112} S_{122} S_{133} S_{233}+6 S_{112} S_{122} S_{123} S_{333}-6 S_{112} S_{113} S_{223} S_{233}+6 S_{112} S_{113} S_{222} S_{333}+ \\
6 S_{112}^{2} S_{233}^{2}-6 S_{112}^{2} S_{223} S_{333}-6 S_{111} S_{133} S_{223}^{2}+6 S_{111} S_{133} S_{222} S_{233}+6 S_{111} S_{123} S_{223} S_{233}-6 S_{111} S_{123} S_{222} S_{333}- \\
6 S_{111} S_{122} S_{233}^{2}+6 S_{111} S_{122} S_{223} S_{333} \\
I_{6}=48 S_{123}^{6}-144 S_{122} S_{123}^{4} S_{133}+144 S_{122}^{2} S_{123}^{2} S_{133}^{2}-48 S_{122}^{3} S_{133}^{3}+72 S_{113} S_{123}^{3} S_{133} S_{222}-144 S_{113} S_{123}^{4} S_{223}- \\
72 S_{113} S_{122} S_{123} S_{133}^{2} S_{222}+72 S_{113} S_{122} S_{123}^{2} S_{133} S_{223}+216 S_{113} S_{122} S_{123}^{3} S_{233}+72 S_{113} S_{122}^{2} S_{133}^{2} S_{223}- \\
216 S_{113} S_{122}^{2} S_{123} S_{133} S_{233}-72 S_{113} S_{122}^{2} S_{123}^{2} S_{333}+72 S_{113} S_{122}^{3} S_{133} S_{333}+18 S_{113}^{2} S_{133}^{2} S_{222}^{2}-
\end{gathered}
$$




$$
\begin{aligned}
& 72 S_{113}^{2} S_{123} S_{133} S_{222} S_{223}+144 S_{113}^{2} S_{123}^{2} S_{223}^{2}-72 S_{113}^{2} S_{123}^{2} S_{222} S_{233}+72 S_{113}^{2} S_{122} S_{133} S_{223}^{2}- \\
& 36 S_{113}^{2} S_{122} S_{133} S_{222} S_{233}-216 S_{113}^{2} S_{122} S_{123} S_{223} S_{233}+144 S_{113}^{2} S_{122} S_{123} S_{222} S_{333}+162 S_{113}^{2} S_{122}^{2} S_{233}^{2}- \\
& 144 S_{113}^{2} S_{122}^{2} S_{223} S_{333}-48 S_{113}^{3} S_{223}^{3}+72 S_{113}^{3} S_{222} S_{223} S_{233}-24 S_{113}^{3} S_{222}^{2} S_{333}-72 S_{112} S_{123}^{2} S_{133}^{2} S_{222}+ \\
& 216 S_{112} S_{123}^{3} S_{133} S_{223}-144 S_{112} S_{123}^{4} S_{233}+72 S_{112} S_{122} S_{133}^{3} S_{222}-216 S_{112} S_{122} S_{123} S_{133}^{2} S_{223}+ \\
& 72 S_{112} S_{122} S_{123}^{2} S_{133} S_{233}+72 S_{112} S_{122} S_{123}^{3} S_{333}+72 S_{112} S_{122}^{2} S_{133}^{2} S_{233}-72 S_{112} S_{122}^{2} S_{123} S_{133} S_{333}- \\
& 36 S_{112} S_{113} S_{133}^{2} S_{222} S_{223}-216 S_{112} S_{113} S_{123} S_{133} S_{223}^{2}+360 S_{112} S_{113} S_{123} S_{133} S_{222} S_{233}+72 S_{112} S_{113} S_{123}^{2} S_{223} S_{233}- \\
& 216 S_{112} S_{113} S_{123}^{2} S_{222} S_{333}+36 S_{112} S_{113} S_{122} S_{133} S_{223} S_{233}-108 S_{112} S_{113} S_{122} S_{133} S_{222} S_{333}- \\
& 216 S_{112} S_{113} S_{122} S_{123} S_{233}^{2}+360 S_{112} S_{113} S_{122} S_{123} S_{223} S_{333}-36 S_{112} S_{113} S_{122}^{2} S_{233} S_{333}+72 S_{112} S_{113}^{2} S_{223}^{2} S_{233}- \\
& 144 S_{112} S_{113}^{2} S_{222} S_{233}^{2}+72 S_{112} S_{113}^{2} S_{222} S_{223} S_{333}+162 S_{112}^{2} S_{133}^{2} S_{223}^{2}-144 S_{112}^{2} S_{133}^{2} S_{222} S_{233}- \\
& 216 S_{112}^{2} S_{123} S_{133} S_{223} S_{233}+144 S_{112}^{2} S_{123} S_{133} S_{222} S_{333}+144 S_{112}^{2} S_{123}^{2} S_{233}^{2}-72 S_{112}^{2} S_{123}^{2} S_{223} S_{333}+ \\
& 72 S_{112}^{2} S_{122} S_{133} S_{233}^{2}-36 S_{112}^{2} S_{122} S_{133} S_{223} S_{333}-72 S_{112}^{2} S_{122} S_{123} S_{233} S_{333}+18 S_{112}^{2} S_{122}^{2} S_{333}^{2}+ \\
& 72 S_{112}^{2} S_{113} S_{223} S_{233}^{2}-144 S_{112}^{2} S_{113} S_{223}^{2} S_{333}+72 S_{112}^{2} S_{113} S_{222} S_{233} S_{333}-48 S_{112}^{3} S_{233}^{3}+72 S_{112}^{3} S_{223} S_{233} S_{333}- \\
& 24 S_{112}^{3} S_{222} S_{333}^{2}-24 S_{111} S_{133}^{3} S_{222}^{2}+144 S_{111} S_{123} S_{133}^{2} S_{222} S_{223}-72 S_{111} S_{123}^{2} S_{133} S_{223}^{2}- \\
& 216 S_{111} S_{123}^{2} S_{133} S_{222} S_{233}+72 S_{111} S_{123}^{3} S_{223} S_{233}+120 S_{111} S_{123}^{3} S_{222} S_{333}-144 S_{111} S_{122} S_{133}^{2} S_{223}^{2}+ \\
& 72 S_{111} S_{122} S_{133}^{2} S_{222} S_{233}+360 S_{111} S_{122} S_{123} S_{133} S_{223} S_{233}-72 S_{111} S_{122} S_{123} S_{133} S_{222} S_{333}-72 S_{111} S_{122} S_{123}^{2} S_{233}^{2}- \\
& 216 S_{111} S_{122} S_{123}^{2} S_{223} S_{333}-144 S_{111} S_{122}^{2} S_{133} S_{233}^{2}+72 S_{111} S_{122}^{2} S_{133} S_{223} S_{333}+144 S_{111} S_{122}^{2} S_{123} S_{233} S_{333}- \\
& 24 S_{111} S_{122}^{3} S_{333}^{2}+72 S_{111} S_{113} S_{133} S_{223}^{3}-108 S_{111} S_{113} S_{133} S_{222} S_{223} S_{233}+36 S_{111} S_{113} S_{133} S_{222}^{2} S_{333}- \\
& 72 S_{111} S_{113} S_{123} S_{223}^{2} S_{233}+144 S_{111} S_{113} S_{123} S_{222} S_{233}^{2}-72 S_{111} S_{113} S_{123} S_{222} S_{223} S_{333}-36 S_{111} S_{113} S_{122} S_{223} S_{233}^{2}+ \\
& 72 S_{111} S_{113} S_{122} S_{223}^{2} S_{333}-36 S_{111} S_{113} S_{122} S_{222} S_{233} S_{333}-36 S_{111} S_{112} S_{133} S_{223}^{2} S_{233}+72 S_{111} S_{112} S_{133} S_{222} S_{233}^{2}- \\
& 36 S_{111} S_{112} S_{133} S_{222} S_{223} S_{333}-72 S_{111} S_{112} S_{123} S_{223} S_{233}^{2}+144 S_{111} S_{112} S_{123} S_{223}^{2} S_{333}- \\
& 72 S_{111} S_{112} S_{123} S_{222} S_{233} S_{333}+72 S_{111} S_{112} S_{122} S_{233}^{3}-108 S_{111} S_{112} S_{122} S_{223} S_{233} S_{333}+36 S_{111} S_{112} S_{122} S_{222} S_{333}^{2}+ \\
& 18 S_{111}^{2} S_{223}^{2} S_{233}^{2}-24 S_{111}^{2} S_{223}^{3} S_{333}-24 S_{111}^{2} S_{222} S_{233}^{3}+36 S_{111}^{2} S_{222} S_{223} S_{233} S_{333}-6 S_{111}^{2} S_{222}^{2} S_{333}^{2}
\end{aligned}
$$

The algebraic discriminant $D_{3 \mid 3}$, just like any other $S L(3)$-invariant function of $S$, is a function of $I_{4}$ and $I_{6}$ :

$$
D_{3 \mid 3}=32 I_{4}^{3}+3 I_{6}^{2}
$$

When expanded, discriminant $D_{3 \mid 3}$ contains 2040 monomials. See the appendix of the book version of [2], where it is written explicitly for curiosity. Formula (32) is a remarkably concise expression of this disriminant through a pair of invariants, given by beautiful diagrams Fig.13 and Fig.14. It is interesting to extend this type of formulas - eqs. (13), (15), (26) and (32) - to higher $n$ and $r$.

Integral discriminant. Similarly, the integral discriminant is a function of $I_{4}, I_{6}$ :

$$
J_{3 \mid 3}=F\left(I_{4}, I_{6}\right)
$$

where the function $F$ is to be determined. The homogeneity condition does not allow to find $F$ unambigously, it constrains only the overall scaling factor, but not the non-trivial dependence on the ratio of invariants:

$$
F\left(I_{4}, I_{6}\right)=I_{4}^{-1 / 4} G\left(\frac{I_{6}^{2}}{I_{4}^{3}}\right)
$$

To find the function $G(z)$ in this ansatz, we need to use the Ward identities (8). 
Ward identities. Applied to the present case of $n=3, r=3$, the system (8) takes the form

$$
\begin{array}{ll}
\left(\frac{\partial^{2}}{\partial s_{300} \partial s_{102}}-\frac{\partial^{2}}{\partial s_{201} \partial s_{201}}\right) J_{3 \mid 3}=0 & \left(\frac{\partial^{2}}{\partial s_{300} \partial s_{120}}-\frac{\partial^{2}}{\partial s_{210} \partial s_{210}}\right) J_{3 \mid 3}=0 \\
\left(\frac{\partial^{2}}{\partial s_{300} \partial s_{111}}-\frac{\partial^{2}}{\partial s_{201} \partial s_{210}}\right) J_{3 \mid 3}=0 & \left(\frac{\partial^{2}}{\partial s_{300} \partial s_{111}}-\frac{\partial^{2}}{\partial s_{210} \partial s_{201}}\right) J_{3 \mid 3}=0 \\
\left(\frac{\partial^{2}}{\partial s_{300} \partial s_{003}}-\frac{\partial^{2}}{\partial s_{201} \partial s_{102}}\right) J_{3 \mid 3}=0 & \left(\frac{\partial^{2}}{\partial s_{300} \partial s_{030}}-\frac{\partial^{2}}{\partial s_{210} \partial s_{120}}\right) J_{3 \mid 3}=0 \\
\left(\frac{\partial^{2}}{\partial s_{300} \partial s_{012}}-\frac{\partial^{2}}{\partial s_{201} \partial s_{111}}\right) J_{3 \mid 3}=0 & \left(\frac{\partial^{2}}{\partial s_{300} \partial s_{021}}-\frac{\partial^{2}}{\partial s_{210} \partial s_{111}}\right) J_{3 \mid 3}=0 \\
\left(\frac{\partial^{2}}{\partial s_{300} \partial s_{012}}-\frac{\partial^{2}}{\partial s_{210} \partial s_{102}}\right) J_{3 \mid 3}=0 & \left(\frac{\partial^{2}}{\partial s_{300} \partial s_{021}}-\frac{\partial^{2}}{\partial s_{201} \partial s_{120}}\right) J_{3 \mid 3}=0
\end{array}
$$

where $s$-parameters and $S$-parameters are related by

$$
\begin{gathered}
s_{300}=S_{111}, \quad s_{210}=3 S_{112}, \quad s_{201}=3 S_{113}, \quad s_{030}=S_{222}, \quad s_{120}=3 S_{122}, \quad s_{021}=3 S_{223} \\
s_{003}=S_{333}, \quad s_{102}=3 S_{133}, \quad s_{012}=3 S_{233}, \quad s_{111}=6 S_{123}
\end{gathered}
$$

Particular equations in this system are, of course, not $S L(3)$ invariant.

Invariant form of Ward identities. To rewrite the above differential equations in $S L(3)$-invariant form, we use again the method of invariant differential operators - sum the Ward operators with appropriate $S$ dependent coefficients to form an invariant operator $\hat{O}_{4}$, given by the diagram at Fig.15. The antisymmetrized combination of two derivatives (two black rhombuses) which is a part of this diagram, ensures that $\hat{O}_{4}$ is a

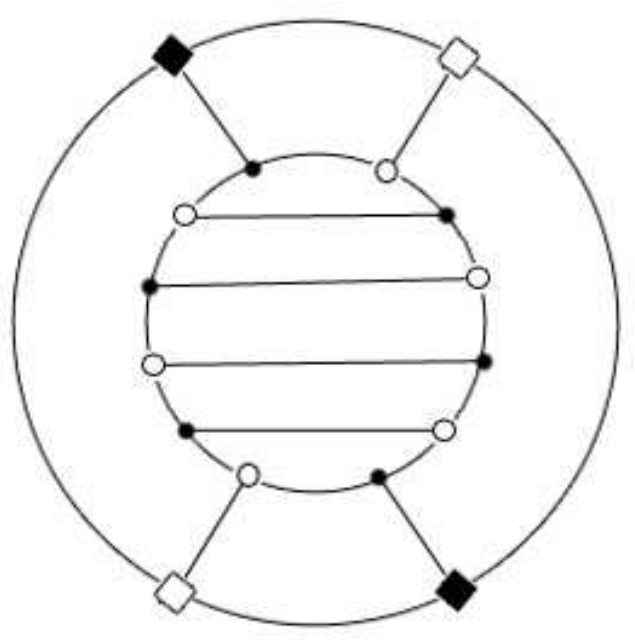

Figure 15: Invariant differential operator $\hat{O}_{4}$ for the case $3 \mid 3$, represented as a diagram of tensor contraction. Black 3-valent vertices represent tensor $S$, white 3 -valent vertices represent tensor $\epsilon$, black 3 -valent rhombuses represent tensor $\partial / \partial S$, white 3 -valent rhombuses represent tensor $\epsilon_{*}$. 
linear combination of Ward operators and annihilates $J_{3 \mid 3}$. Operator $\hat{O}_{4}$ contains 4 horizontal lines in the inner circle and belongs to an infinite family of operators with $2 p$ horizontal lines in the inner circle:

$$
\hat{O}_{2 p}, \quad p=1,2,3, \ldots
$$

of which the simplest are $\hat{O}_{0}$ and $\hat{O}_{2}$, given by diagrams at Fig.16. However, operators $\hat{O}_{0}$ and $\hat{O}_{2}$ are too simple: in fact, they do not constrain $J_{3 \mid 3}$ at all. Thus $\hat{O}_{4}$ is the main operator we use in this section.


Figure 16: Invariant differential operators $\hat{O}_{0}$ and $\hat{O}_{2}$ for the case $3 \mid 3$, represented as diagrams of tensor contraction. Black 3 -valent vertices represent tensor $S$, white 3 -valent vertices represent tensor $\epsilon$, black 3 -valent rhombuses represent tensor $\partial / \partial S$, white 3 -valent rhombuses represent tensor $\epsilon_{*}$. These operators cannot be used to find $J_{3 \mid 3}$, because they do not give any non-trivial equation on it.

The action of $\hat{O}_{4}$ on invariants and their products is given by

$$
\begin{gathered}
\hat{O}_{4}\left(\begin{array}{c}
I_{4} \\
I_{6}
\end{array}\right)=\left(\begin{array}{c}
-\frac{140}{9} I_{4}^{2} \\
-\frac{98}{3} I_{4} I_{6}
\end{array}\right) \\
\hat{O}_{4}\left(\begin{array}{cc}
I_{4}^{2} & I_{4} I_{6} \\
I_{4} I_{6} & I_{6}^{2}
\end{array}\right)=\left(\begin{array}{cc}
\frac{1}{3} I_{6}^{2}-\frac{472}{9} I_{4}^{3} & -\frac{770}{9} I_{4}^{2} I_{6} \\
-\frac{770}{9} I_{4}^{2} I_{6} & \frac{256}{3} I_{4}^{4}-\frac{340}{3} I_{6}^{2} I_{4}
\end{array}\right)
\end{gathered}
$$

Applying (28), we obtain

$\hat{O}_{4} F\left(I_{4}, I_{6}\right)=-\frac{140}{9} I_{4}^{2} \frac{\partial F}{\partial I_{4}}-\frac{98}{3} I_{4} I_{6} \frac{\partial F}{\partial I_{6}}+\left(\frac{1}{6} I_{6}^{2}-\frac{32}{9} I_{4}^{3}\right) \frac{\partial^{2} F}{\partial I_{4}^{2}}-\frac{112}{3} I_{4}^{2} I_{6} \frac{\partial^{2} F}{\partial I_{4} \partial I_{6}}+\left(\frac{128}{3} I_{4}^{4}-24 I_{4} I_{6}^{2}\right) \frac{\partial^{2} F}{\partial I_{6}^{2}}$ 
For the integral discriminant, this expression vanishes. Substituting the ansatz (33) and making the necessary algebraic transformations, we obtain a differential equation on the function $G(z)$ :

$$
\left(144 z^{2}+1536 z\right) \frac{\partial^{2} G(z)}{\partial z^{2}}+(216 z+768) \frac{\partial G(z)}{\partial z}+5 G(z)=0
$$

which is nothing but Gauss hypergeometric equation

$$
t(1-t) \frac{\partial^{2} G(t)}{\partial t^{2}}+(c-(a+b+1) t) \frac{\partial G(t)}{\partial t}-a b G(t)=0
$$

with $a=1 / 12, b=5 / 12, c=1 / 2$ and $t=-3 / 32 z$. This is the invariant form of $n=3, r=3$ Ward identities.

Solution. In terms of the Gauss hypergeometric function, the integral discriminant equals

$$
J_{3 \mid 3}(S)=c_{1} \cdot I_{4}^{-1 / 4}{ }_{2} F_{1}\left(\left[\frac{1}{12}, \frac{5}{12}\right],\left[\frac{1}{2}\right],-\frac{3 I_{6}^{2}}{32 I_{4}^{3}}\right)+c_{2} \cdot I_{6} I_{4}^{-7 / 4}{ }_{2} F_{1}\left(\left[\frac{7}{12}, \frac{11}{12}\right],\left[\frac{3}{2}\right],-\frac{3 I_{6}^{2}}{32 I_{4}^{3}}\right)
$$

where $c_{1,2}$ are the two constants, parametrising the general solution of Ward identities. Particular solutions

$$
J_{3 \mid 3}^{(1)}(S)=I_{4}^{-1 / 4}{ }_{2} F_{1}\left(\left[\frac{1}{12}, \frac{5}{12}\right],\left[\frac{1}{2}\right],-\frac{3 I_{6}^{2}}{32 I_{4}^{3}}\right) \quad \text { and } \quad J_{3 \mid 3}^{(2)}(S)=I_{6} I_{4}^{-7 / 4}{ }_{2} F_{1}\left(\left[\frac{7}{12}, \frac{11}{12}\right],\left[\frac{3}{2}\right],-\frac{3 I_{6}^{2}}{32 I_{4}^{3}}\right)
$$

are associated with different integration contours and can be called the first and the second branches of $J_{3 \mid 3}$. One can see, that the results of this section are parallel to those of sec.3.2 - even the rational parameters in the hypergeometric function are the same.

Singularities. Notice, that the point $t=1$ corresponds to

$$
-\frac{3 I_{6}^{2}}{32 I_{4}^{3}}=1
$$

which is just the discriminant's vanishing locus $32 I_{4}^{3}+3 I_{6}^{2}=D_{3 \mid 3}=0$. To investigate the two other suspicious points $t=0$ and $t=\infty$, let us study asymptotics at these points. Using (19), we find

$$
\begin{array}{cc}
J_{3 \mid 3}^{(1)}(S) \sim I_{4}^{-1 / 4}+O(t), \quad \text { when } t \rightarrow 0 \\
J_{3 \mid 3}^{(2)}(S) \sim I_{6} I_{4}^{-7 / 4}+O(t), \quad \text { when } t \rightarrow 0
\end{array}
$$

Using (20), we find

$$
\begin{gathered}
J_{3 \mid 3}^{(1)}(S) \sim \frac{-\Gamma(1 / 2)}{\Gamma(1 / 12) \Gamma(5 / 12)} I_{4}^{-1 / 4} \log \left(1+\frac{3 I_{6}^{2}}{32 I_{4}^{3}}\right)+O\left(t^{0}\right), \quad \text { when } t \rightarrow 1 \\
J_{3 \mid 3}^{(2)}(S) \sim \frac{-\Gamma(3 / 2)}{\Gamma(7 / 12) \Gamma(11 / 12)} I_{6} I_{4}^{-7 / 4} \log \left(1+\frac{3 I_{6}^{2}}{32 I_{4}^{3}}\right)+O\left(t^{0}\right), \quad \text { when } t \rightarrow 1
\end{gathered}
$$


Using (19) and (21), we find

$$
\begin{array}{ll}
J_{3 \mid 3}^{(1)}(S) \sim \frac{\Gamma(1 / 2) \Gamma(1 / 3)}{\Gamma(5 / 12)^{2}}\left(\frac{3}{32}\right)^{-1 / 12} I_{6}^{-1 / 6}+O\left(t^{-5 / 12}\right), & \text { when } t \rightarrow \infty \\
J_{3 \mid 3}^{(2)}(S) \sim \frac{\Gamma(1 / 3) \Gamma(3 / 2)}{\Gamma(11 / 12)^{2}}\left(\frac{3}{32}\right)^{-7 / 12} I_{6}^{-1 / 6}+O\left(t^{-11 / 12}\right), & \text { when } t \rightarrow \infty
\end{array}
$$

We can see from these results, that singularities of the integral discriminant $J_{3 \mid 3}$ at finite values of $I_{4}, I_{6}$ are completely controlled by the algebraic discriminant $D_{3 \mid 3}$. The singularity at discriminant locus is logarithmic. Other singularities, not related to the algebraic discriminant, are situated at infinite values of invariants: $I_{4} \rightarrow \infty(t \rightarrow 0)$ and $I_{6} \rightarrow-\infty(t \rightarrow \infty)$.

Hypergeometric integral. Using eq. (22) once again, we obtain

$$
J_{3 \mid 3}(S)=c_{1} \cdot \int_{0}^{1} d s \frac{s^{-7 / 12}(1-s)^{-11 / 12}}{\left(32 I_{4}^{3}+3 s I_{6}^{2}\right)^{1 / 12}}+c_{2} I_{6} \cdot \int_{0}^{1} d s \frac{s^{-1 / 12}(1-s)^{-5 / 12}}{\left(32 I_{4}^{3}+3 s I_{6}^{2}\right)^{7 / 12}}
$$

Just as in the case of $J_{2 \mid 4}$, it is possible to make the transformation (25), which gives

$$
\begin{gathered}
J_{3 \mid 3}^{(1)}(S)=\left(D_{3 \mid 3}\right)^{-1 / 12}{ }_{2} F_{1}\left(\left[\frac{1}{12}, \frac{1}{12}\right],\left[\frac{1}{2}\right], \frac{3 I_{6}^{2}}{32 D_{3 \mid 3}}\right) \\
J_{3 \mid 3}^{(2)}(S)=I_{6}\left(D_{3 \mid 3}\right)^{-7 / 12}{ }_{2} F_{1}\left(\left[\frac{7}{12}, \frac{7}{12}\right],\left[\frac{3}{2}\right], \frac{3 I_{6}^{2}}{32 D_{3 \mid 3}}\right)
\end{gathered}
$$

We conclude, that cases $2 \mid 4$ and $3 \mid 3$ literally correspond one to another. From the point of view of discriminant theory, this is a remarkable correspondence: discriminant $D_{2 \mid 4}$ is a simple two-dimensional discriminant, which is well-known and studied, while $D_{3 \mid 3}$ is a three-dimensional discriminant, much more complicated and less widely known. The study of integral discriminants reveals a parallel between these two cases.

\section{Conclusion}

In this paper we have described the first steps into the study of non-Gaussian averages

$$
J_{n \mid r}(S)=\int d x_{1} \ldots d x_{n} e^{-S\left(x_{1}, \ldots, x_{n}\right)}=\text { function of invariants of } S
$$

which are functions only of $S L(n)$ invariants of $S$, because of inherent $S L(n)$ symmetry. The real motivation of this study is generalisation of the well-known Gaussian formula

$$
J_{n \mid 2}(S)=\int d x_{1} \ldots d x_{n} e^{-S_{i j} x_{i} x_{j}}=\frac{1}{\sqrt{\operatorname{det} S}}
$$

to non-quadratic forms $S$. If found, such generalisation may immediately have a wide range of applications in 
statistical physics and quantum field theory. We have worked out several low-dimensional cases and present the results in the form of the following table:

\begin{tabular}{l|c|c|c|l}
$n$ & $r$ & Invariants & Discriminant $D_{n \mid r}$ & Integral discriminant $J_{n \mid r}$ \\
\hline 2 & 2 & $I_{2}$ & $I_{2}$ & $I_{2}^{-1 / 2}$ \\
2 & 3 & $I_{4}$ & $I_{2}$ & $I_{4}^{-1 / 6}$ \\
2 & 4 & $I_{2}, I_{3}$ & $I_{2}^{3}-6 I_{3}^{2}$ & $I_{2}^{-1 / 4} \cdot \sum_{i=0}^{\infty} \frac{1}{i !} \cdot \frac{(1 / 12)_{i}(5 / 12)_{i}}{(1 / 2)_{i}} \cdot\left(\frac{6 I_{3}^{2}}{I_{2}^{3}}\right)^{i}$ \\
2 & 5 & $I_{4}, I_{8}, I_{12}$ & $I_{4}^{2}-64 I_{8}$ & $I_{4}^{-1 / 10} \cdot \sum_{i, j=0}^{\infty} \frac{1}{i ! j !} \cdot \frac{(3 / 10)_{i+j}(1 / 10)_{2 i+3 j}(1 / 10)_{j}}{(2 / 5)_{i+2 j}(3 / 5)_{i+2 j}} \cdot\left(\frac{16 I_{8}}{I_{4}^{2}}\right)^{i}\left(\frac{128 I_{12}}{3 I_{4}^{3}}\right)^{j}$ \\
3 & 2 & $I_{3}$ & $I_{3}$ & $I_{3}^{-1 / 2}$ \\
3 & 3 & $I_{4}, I_{6}$ & $32 I_{4}^{3}+3 I_{6}^{2}$ & $I_{4}^{-1 / 4} \cdot \sum_{i=0}^{\infty} \frac{1}{i !} \cdot \frac{(1 / 12)_{i}(5 / 12)_{i}}{(1 / 2)_{i}} \cdot\left(-\frac{3 I_{6}^{2}}{32 I_{4}^{3}}\right)^{i}$ \\
& & & $\ldots$ & $\ldots$
\end{tabular}

where

$$
(a)_{k} \equiv \frac{\Gamma(a+k)}{\Gamma(a)}=a(a+1) \cdots(a+k-1)
$$

As one can see, these series posess a nice structure: they are all hypergeometric, i.e, their coefficients are ratios of $\Gamma$-functions. Despite there are too few examples to make far-going conclusions, we conjecture that

$$
J_{n \mid r}(S)=\int d x_{1} \ldots d x_{n} e^{-S\left(x_{1}, \ldots, x_{n}\right)}=\text { hypergeometric function of invariants of } S
$$

To support this conjecture, it is necessary at least to calculate a few more examples. As follows from the table Fig. 3, the next simplest examples would be $J_{2 \mid 6}, J_{3 \mid 4}$ and $J_{4 \mid 3}$. Also, because of hypergeometric nature of integral discriminants, it is interesting to find their $q$-deformation, as well as the CFT representations of these objects, in the spirit of [13]. Especially interesting is the interplay between non-trivial $q$-deformation and action-independence of integral discriminants. Of course, most interesting would be generalization of our results to functional integrals, some independent attempts in this direction have already been made [14].

\section{Acknowledgements}

We are indebted to V.Dolotin and V.Tkachev for stimulating discussions. Our work is partly supported by Russian Federal Nuclear Energy Agency and the Russian President's Grant of Support for the Scientific Schools NSh-3035.2008.2, by RFBR grant 07-02-00547, by the joint grants 09-01-92440-CE, 09-02-91005-ANF, 09-0293105-CNRS and by the NWO project 047.011.2004.026. The work of Sh.Shakirov is also supported in part by the Moebius Contest Foundation for Young Scientists and by the Dynasty Foundation. 


\section{References}

[1] V.Dolotin, QFT's With Action of Degree 3 and Higher and Degeneracy of Tensors, hep-th/9706001

[2] V.Dolotin and A.Morozov, Introduction to Non-Linear Algebra, World Scientific, 2007, hep-th/0609022

[3] N. Beklemishev, Invariants of cubic forms of four variables, Moscow Univ.Bull. 37 (1982) 54-62;

D. Hilbert, B. Sturmfels and R. Laubenbacher, Theory of algebraic invariants, Cambridge University Press (1993);

H. Derksen and G. Kemper, Computational invariant theory. Invariant Theory and Algebraic Transformation Groups, Springer-Verlag, Berlin, encyclopaedia of Mathematical Sciences 130 (2002);

B.Sturmfels, Algorithms in invariant theory, Springer-Verlag, New York (2008)

[4] I.M. Gelfand, M.M. Kapranov, and A.V. Zelevinsky, Discriminants, Resultants and Multidimensional Determinants, Birkhauser, 1994

[5] V.Dolotin, On discriminants of polylinear forms, arXiv:alg-geom/9511010; On invariant theory. alggeom/9512011;

Yu. Chernyakov and V. Dolotin, Operations and Identities in Tensor Algebra, arXiv:math/0501206

[6] D. Manocha and J.Canny, Multipolynomial Resultant Algorithms, J.Symb.Comp. 15 (1993) 99 - 122;

C. Andrea, A.Dickenstein, Explicit formulas for the multivariate resultant, arXiv:math/0007036;

D. Eisenbud and F.O. Schreyer, Resultants and Chow forms via exterior syzygies, J. Amer. Math. Soc.

16 (2003) 537-579, arXiv:math/0111040;

V. Tapia, Polynomial identities for hypermatrices, math-ph/0208010; Invariant and polynomial identities for higher rank matrices, math-ph/0702001;

Sh.Shakirov, Higher discriminants of polynomials, Theor.Math.Phys. 153(2) (2007) 1477, math/0609524;

B. Gustafsson and V.Tkachev, The resultant on compact Riemann surfaces, arXiv:math/0710.2326, Comm. Math. Phys. 286 (2009) 313-358;

A.Morozov and Sh.Shakirov, Analogue of the identity LogDet = TraceLog for resultants, arXiv:0804.4632; Resultants and Contour Integrals, arXiv:0807.4539;

A.Anokhina, A.Morozov and Sh.Shakirov, Resultant as Determinant of Koszul Complex, arXiv:0812.5013

[7] A.Gerasimov, A.Morozov and K.Selivanov, Bogolubov's Recursion and Integrability of Effective Actions, Int.J.Mod.Phys. A16 (2001) 1531-1558, hep-th/0005053;

A. Morozov and A. Niemi, Can Renormalization Group Flow End in a Big Mess? Nucl.Phys. B666 (2003) 311-336, arXiv:hep-th/0304178;

V. Dolotin and A. Morozov, The Universal Mandelbrot Set. Beginning of the Story, arXiv:hep-th/0501235; Int.J.Mod.Phys. A23 (2008) 3613-3684, arXiv:hep-th/0701234;

A. Morozov and M. Serbyn, Non-Linear Algebra and Bogolubov's Recursion, Theor.Math.Phys. 154 (2008) 270-293, hep-th/0703258;

An. Morozov, Universal Mandelbrot Set as a Model of Phase Transition Theory, JETP Lett. 86 (2007) 745-748, arXiv:nlin/0710.2315

[8] M. Penkava and L. Weldon, Infinity Algebras, Massey Products, and Deformations, math/9808058;

B. Keller, Introduction to A-infinity algebras and modules, math/9910179;

A. Tomasiello, A-infinity structure and superpotentials, hep-th/0107195;

A.Losev, Lectures at Dombai and Dubna Schools, 2004;

A.Kozak, Discretization of Chern-Simons-like Theories, 2004-2006, unpublished;

P.Mnev, Notes on Symplicial BF Theory, hep-th/0610326; Discrete BF Theory, arXiv:0809.1160; 
V.Dolotin, A.Morozov and Sh.Shakirov, $A_{\infty}$-structure on simplicial complexes, arXiv:0704.2609;

Higher Nilpotent Analogues of $A_{\infty}$ Structure, Phys.Lett. B651 (2007) 71-73, arXiv:0704.2884

[9] I.M. Gelfand, M.M. Kapranov and A.V. Zelevinsky, Generalized Euler Integrals and A-Hypergeometric Functions, Advances in Math. 84 (1990), 255-271;

I.M. Gelfand, M.M. Kapranov and A.V. Zelevinsky, Hypergeometric Functions, Toric Varieties and Newton Polyhedra in Special Functions, M. Kashiwara, T. Miwa (eds.), ICM-90 Satellite Conference Proceedings, Springer-Verlag Tokyo, 1991;

M. Passare and A. Tsikh, Algebraic Equations and Hypergeometric Series, in: The Legacy of Niels Henrik Abel, O. A. Laudal and R. Piene (eds.), Springer Verlag, Berlin, 2004;

M. Passare, T. Sadykov and A. Tsikh, Singularities of Nonconfluent Hypergeometric Functions in Several Variables, arXiv:math/0405259;

J. Stienstra, GKZ Hypergeometric Structures, math/0511351

[10] A.Morozov, Integrability and Matrix Models, Phys.Usp. 37 (1994) 1-55, hep-th/9303139; Matrix Models as Integrable Systems, hep-th/9502091;

A.Alexandrov, A.Mironov and A.Morozov, Solving Virasoro Constraints in Matrix Models, Fortsch.Phys. 53 (2005) 512-521, hep-th/0412205;

A.Mironov, Matrix Models vs. Matrix Integrals, Theor.Math.Phys. 146 (2006) 63-72, hep-th/0506158;

S. Uhlmann, R. Meinel and A. Wipf, Ward Identities for Invariant Group Integrals, hep-th/0611170

[11] W.Lerche, D.Smit and N. Warner, Differential Equations for Periods and Flat Coordinates in Two Dimensionsional Topological Matter Theories, Nucl.Phys. B372 (1992) 87-112, hep-th/9108013;

A. Ceresole, R. D'Auria, S. Ferrara, W. Lerche and J. Louis, Picard-Fuchs Equations and Special Geometry, Int.J.Mod.Phys. A8 (1993) 79-114, hep-th/9204035;

A.Klemm, W.Lerche and S.Theisen, Nonperturbative Effective Actions of $N=2$ Supersymmetric Gauge Theories, hep-th/9505150;

M.Matone, Instantons and Recursion Relation in $N=2$ Gauge Theory, hep-th/9506102; Koebe 1/4Theorem and Inequalities in $N=2$ Super-QCD, hep-th/9506181;

S.Kachru, A.Klemm, W.Lerche, P.Mayr and C.Vafa, Nonperturbative Results on the Point-Particle Limit of $N=2$ Heterotic String Compactification, hep-th/9508155;

H. Itoyama and A. Morozov, Prepotential and the Seiberg-Witten Theory, Nucl.Phys. B491 (1997) 529573 , hep-th/9512161;

M. Alishahiha, On the Picard-Fuchs equations of the SW models, Phys.Lett. B398 (1997) 100-103, hepth/9609157;

J. Isidro, Integrability, Seiberg-Witten Models and Picard-Fuchs Equations, JHEP 0101 (2001) 043, hepth/0011253

[12] M. Abramowitz and I. Stegun, Handbook of Mathematical Functions with Formulas, Graphs, and Mathematical Tables, 9th printing. Dover, New York, (1972);

I. Gradstein and I. Ryzhik, Tables of Integrals, Series and Products, New York: Academic (1980)

[13] A.Morozov and L.Vinet, q-Hypergeometric Functions in the Formalism of Free Fields, Mod.Phys.Lett. A8 (1993) 2891-2902, arXiv:hep-th/9309026

[14] J. Bohacik and P. Presnajder, Functional integral with phi $i^{4}$ term in the action beyond standard perturbative methods, hep-th/0503235; Nonperturbative approach to(Wiener) functional integral with $\phi^{4}$ interaction, hep-th/0507129; Functional integral with $\phi^{4}$ term in the action beyond standard perturbative methods II, arXiv:0711.4683 